ARTICLE

https://doi.org/10.1038/s41467-019-08579-2

OPEN

\title{
The Elongator subunit Elp3 is a non-canonical tRNA acetyltransferase
}

\author{
Ting-Yu Lin (1) 1, Nour El Hana Abbassi ${ }^{1,2}$, Karol Zakrzewski ${ }^{1,2}$, Andrzej Chramiec-Głąbik1, \\ Małgorzata Jemioła-Rzemińska ${ }^{3,4}$, Jan Różycki ${ }^{5}$ \& Sebastian Glatt (iD ${ }^{1}$
}

The Elongator complex catalyzes posttranscriptional tRNA modifications by attaching carboxy-methyl $\left(\mathrm{cm}^{5}\right)$ moieties to uridine bases located in the wobble position. The catalytic subunit Elp3 is highly conserved and harbors two individual subdomains, a radical S-adenosyl methionine (rSAM) and a lysine acetyltransferase (KAT) domain. The details of its modification reaction cycle and particularly the substrate specificity of its KAT domain remain elusive. Here, we present the co-crystal structure of bacterial Elp3 (DmcElp3) bound to an acetyl-CoA analog and compare it to the structure of a monomeric archaeal Elp3 from Methanocaldococcus infernus (MinElp3). Furthermore, we identify crucial active site residues, confirm the importance of the extended $\mathrm{N}$-terminus for substrate recognition and uncover the specific induction of acetyl-CoA hydrolysis by different tRNA species. In summary, our results establish the clinically relevant Elongator subunit as a non-canonical acetyltransferase and genuine tRNA modification enzyme.

\footnotetext{
${ }^{1}$ Max Planck Laboratory, Malopolska Centre of Biotechnology (MCB), Jagiellonian University, Krakow 30-387, Poland. ${ }^{2}$ Postgraduate School of Molecular Medicine, Warsaw 02-091, Poland. ${ }^{3}$ Faculty of Biochemistry, Biophysics and Biotechnology, Jagiellonian University, Krakow 30-387, Poland. ${ }^{4}$ Malopolska Centre of Biotechnology (MCB), Jagiellonian University, Krakow 30-387, Poland. ${ }^{5}$ Bionanoscience and Biochemistry Laboratory, Malopolska Centre of Biotechnology (MCB), Jagiellonian University, Krakow 30-387, Poland. Correspondence and requests for materials should be addressed to S.G. (email: sebastian.glatt@uj.edu.pl)
} 
ver 170 different RNA base modifications are currently described ${ }^{1}$ and a majority is found in tRNA molecules of all three domains of life $\mathrm{e}^{2,3}$. The functions of tRNA modifications can be separated into three main categories depending on the modified positions, either (i) stabilizing the structural integrity of the core tRNA fold ${ }^{4}$, (ii) contributing to the correct amino-acylation of respective tRNAs at the acceptor stem loop ${ }^{5}$ or (iii) enhancing the decoding potential and translation fidelity at the ribosome ${ }^{6,7}$. The latter group of tRNA modifications is mostly found around the anticodon stem loop (ASL), particularly at the so-called "hot spot" positions 34 and $37^{2,7,8}$. As modifications in this region can provide additional chemical bonds between the ASL and its cognate and near-cognate codons during the ribosomal decoding process, they are crucial for fine-tuning translation elongation ${ }^{8-11}$ and cotranslational folding dynamics ${ }^{12,13}$. In agreement, the lack of certain uridine modifications in the wobble position $\left(\mathrm{U}_{34}\right)$, such as 5-methoxycarbonylmethyl $\left(\mathrm{mcm}^{5}\right), 5$-carbamoylmethyl $\left(\mathrm{ncm}^{5}\right)$ and 5-methoxy-carbonyl-methyl-2-thio $\left(\mathrm{mcm}^{5} \mathrm{~s}^{2}\right)$, were shown to induce cellular stress ${ }^{14}$, increase intracellular protein aggregation and disturb proteome homeostasis ${ }^{15,16}$.

The eukaryotic Elongator complex was initially described as a transcription-related elongation factor due to its association with hyper-phosphorylated RNA Polymerase $\mathrm{II}^{17}$ and the predicted presence of a potential histone/lysine acetyltransferase (KAT) domain in its catalytic Elp3 subunit ${ }^{18}$. Although some recent reports still follow that initial hypothesis, an increasing number of studies supports the idea that Elongator in fact represents a genuine tRNA modification enzyme, which catalyzes the $\mathrm{cm}^{5} \mathrm{U}_{34}$ modification, representing the first step in a cascade leading to different types of $U_{34}$ modifications ${ }^{19-22}$. The $\mathrm{cm}^{5}$ moiety can be subsequently methylated by the methyl transferase Trm9 resulting in $\mathrm{mcm}^{5} \mathrm{U}_{34}$ ref. ${ }^{23}$. In three yeast tRNAs, namely tRNAGlu $_{U U C}$, tRNA $^{\text {Gln }}{ }_{U U G}$, and tRNA ${ }^{\text {Lys }}$ UUU, this "primal" Elongator modification is succeeded by an additional thiolation ${ }^{24}$ leading to $\mathrm{mcm}^{5} \mathrm{~s}^{2} \mathrm{U}_{34}$ or is converted into $\mathrm{ncm}^{5} \mathrm{U}_{34}$ by a yet unknown mechanism in other tRNA species ${ }^{25}$.

The fully assembled eukaryotic complex consists of two copies of each of its six subunits (Elp1-6), which are arranged in twoindependent modules, the catalytic Elp123 and the associated Elp456 sub-complexes ${ }^{26-28}$. All six Elongator subunits are highly conserved among eukaryotes and the Elp3 subunit is even found in all three domains of life, including all archaea and some bacterial clades ${ }^{20}$. Elp3 acts as the catalytic subunit, but the loss of any of the six subunits results in hypo-modified $U_{34}$ tRNAs in yeast ${ }^{19,29}$, indicating that the complete integrity of the complex is important for its function. The key role of Elongator in maintaining the stringent homeostasis of the cellular proteome explains the pleotropic phenotypes associated with Elp3 deficiency, including neurogenesis ${ }^{30}$, DNA repair ${ }^{31}$, exocytosis ${ }^{32}$, genome demethylation ${ }^{33}$, protein acetylation ${ }^{34,35}$, mitochondria dysfunction $^{36}$, and tRNA modification ${ }^{22}$. Moreover, patientderived mutations and deficiencies in different Elongator subunits are associated with severe human diseases ${ }^{37,38}$, such as cancer $^{39}$ and neurodegenerative diseases ${ }^{40}$, including familial dysautonomia $^{41}$, amyotrophic lateral sclerosis ${ }^{42}$, intellectual disabilities $^{43}$, and ataxia ${ }^{44}$.

Over the last decade, significant progress has been made towards an understanding of the structural and functional rationale behind the modification reaction conducted by the catalytically active Elp3 subunit ${ }^{20,21,45,46}$. We previously determined the high resolution crystal structure of the bacterial Elp3 homologue from Dehalococcoides mccartyi (DmcElp3), revealing the tight interplay between the involved radical $S$-adenosyl methionine (rSAM) and KAT domains. The rSAM domain of Elp3 is highly similar to $\mathrm{RlmN}^{47}$, which also contains a $\mathrm{Fe}-\mathrm{S}$ cluster and methylates rRNAs and tRNAs using its reductive
SAM cleavage activity ${ }^{48}$. The C-terminal KAT domain is highly similar to the known superfamily of GCN5-like acetyl transferases ${ }^{49}$, which bind linear peptides and acetylate target lysines via their acetyl-CoA hydrolysis activity ${ }^{50}$. The two domains form a large domain interface, which blocks the canonical KAT peptide-binding site, and a partially disordered central linker region coordinates a $\mathrm{Zn}$-ion in between $\mathrm{rSAM}$ and KAT. The two domains form a cleft that contains several highly conserved basic residues, which bind and accommodate the tRNA anticodon stem loop (ASL). The current model of a radical based Elp3-mediated $\mathrm{cm}^{5}$-modification reaction is proposed as follows (i) Elp3 recruits SAM and cleaves it to generate a 5'deoxyadenosine radical (5'$\mathrm{dA}$ ) in the rSAM domain, (ii) Elp3 hydrolyzes acetyl-CoA in the KAT domain and (iii) an acetyl radical is formed by the products of the two domains and (iv) is transferred onto the C5 position of $\mathrm{U}_{34}$ in the bound tRNA molecule.

In comparison to the overwhelming amount of recently published phenotypical and clinical studies on the Elongator complex, the number of available structural and biochemical data remain minuscule. However, without a deeper mechanistic knowledge of the underlying mechanisms in different organisms, the genuine cellular function of Elongator and the details of the reaction cycle and its intermediates remain elusive and highly speculative. Here, we present the structure of DmcElp3 bound to an acetyl-CoA derivative that reveals ligand coordination and allows the precise identification of several active site residues. In addition, we present the crystal structure of monomeric Elp3 from archaea and compare it to the previously known dimeric bacterial Elp3 structure. Furthermore, we are able to measure acetyl-CoA hydrolysis rates and tRNA binding activities for different Elp3 homologues. Last but not least, we provide biochemical evidence that specific tRNAs, but neither histone or tubulin peptides nor other nucleic acids, act as the exclusive trigger for acetyl-CoA hydrolysis in bacterial, archaeal and eukaryotic Elp3s. Our results define Elongator and its Elp3 subunit as a genuine tRNA editing enzyme in all three domains of life and resolve the ongoing debate about a direct involvement of Elongator in any cellular activity other than tRNA modification.

\section{Results}

DmcElp3 binds acetyl-CoA like other KAT domain proteins. We have previously determined the crystal structure of Elp3 from Dehalococcoides mccartyi and identified the potential acetyl-CoA binding pocket and a specific blocking loop that seems to functionally link acetyl-CoA- and tRNA-binding ${ }^{21}$. Furthermore, we found that several potent key residues residing in the acetyl-CoA binding pocket (e.g. K77, K193, E386, and Y441) are conserved among various Elp3s and showed that the equivalent single amino acid substitutions in yeast lead to Elongator loss-of-function phenotypes. To obtain detailed structural insights into acetyl-CoA coordination, we combined the respective blocking loop deletion (DmcElp3 $390-406(\mathrm{GSGSG})$ ) with additional active site mutations (e.g. E386A, H388A, R411T) for co-crystallization trials in the presence of acetyl-CoA and different acetyl-CoA analogues. We successfully co-crystallized DmcElp3 $390-406$ (GSGSG)/E386A (Supplementary Fig. 1a) in complex with desulfo-CoA (DmcElp3-DCA) using similar crystallization conditions as for apo DmcElp3. We solved and refined the structure at $2.7 \AA$ resolution using DmcElp3 (PDB ID 5L7L) as search model for molecular replacement (Fig. 1a and Table 1). The overall structure of DmcElp3-DCA closely matches the structure of DmcElp3 (Supplementary Fig. 1b), indicating that ligand binding has very little or no influence on the structural arrangement of the two domains or the $\mathrm{Fe}-\mathrm{S}$ cluster mediated 
a

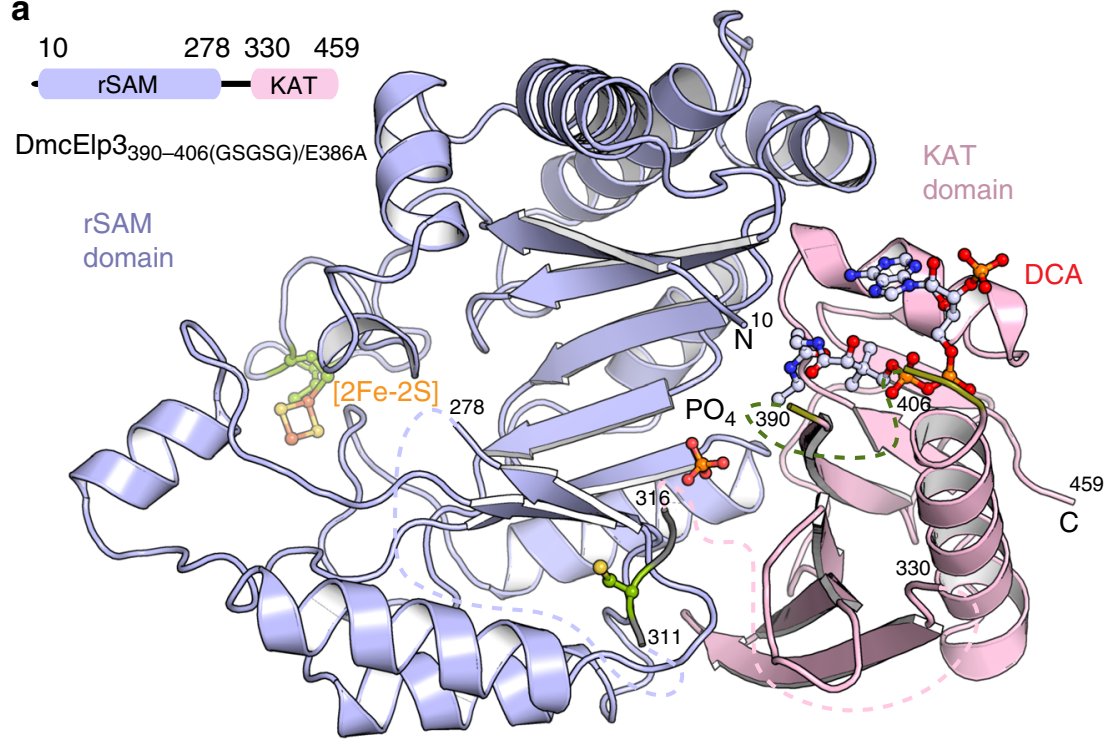

b

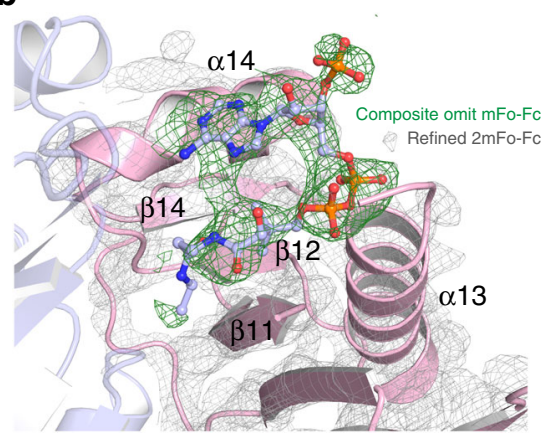

C

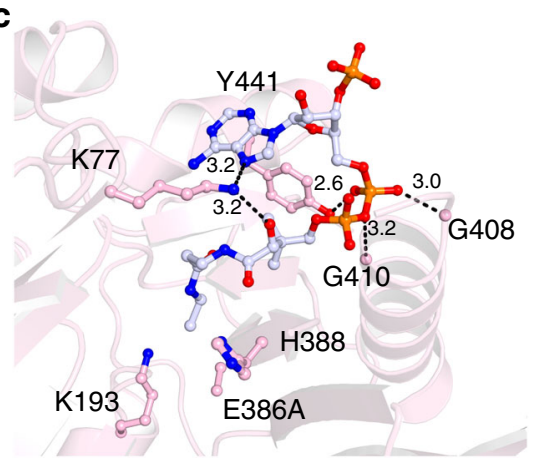

Fig. 1 The crystal structure of DCA bound to DmcElp3390-406(GSGSG)/E386A. a Overall structure of DmcElp3-DCA in cartoon representation. The rSAM domain (blue), KAT domain (pink), and acetyl-CoA blocking loop (olive) are highlighted. Disordered loops are shown using dotted lines. Fe-S cluster binding and zinc binding residues are highlighted in green. Fe-S cluster (orange and yellow spheres) and $\mathrm{PO}_{4}$ (orange and red spheres) are highlighted. Respective amino acid numbers are indicated. $\mathbf{b}$ The Fo-Fc simulated annealing omit map (green) of DCA and the refined 2Fo-Fc map (gray) are shown at $1.5 \sigma$ in the binding pocket of DmcElp3 $390-406$ (GSGSG)/E386A. The model of DmcElp3 is shown in cartoon and the located DCA molecule in ball and sticks representations. c The interaction of DCA and the residues residing in the binding pocket of DmcElp3 $390-406$ (GSGSG)/E386A. Hydrogen bonds are highlighted in dotted lines while bond lengths were analyzed using LigPlot. All involved residues and distance are indicated

rSAM dimerization. This observation is consistent with our previous results from SAXS analyses performed in the presence and absence of acetyl-CoA ${ }^{21}$. The density of DCA is clearly visible in initial, refined and simulated annealing composite omit maps and allowed its unambiguous placement (Fig. 1b). DCA is structurally similar to acetyl-CoA but lacks both, the acetyl group as well as the thiol-moiety, and therefore represents a postcleavage product analogue. DCA binds to the KAT domain of DmcElp3 in a similar way as acetyl-CoA is coordinated by the active site of GCN5 from Tetrahymena (Supplementary Fig. 1b). In detail, the two phosphate groups of DCA are in close proximity to the glycine residues residing at the start of the blocking loop. The rest of DCA, including pantothenic acid and $\beta$-mercaptoethylamine, are pointing deeper into the predicted binding pocket. Furthermore, previously identified conserved residues, including K77, K193, and Y441, are involved in ligand binding and in close proximity to DCA (Fig. 1c and Supplementary Fig. 1c). The $\beta$-mercaptoethylamine part of DCA was not clearly resolved in our densities, presumably because this part of the ligand is flexible in the absence of the missing tRNA substrate. Considering the observation that DCA stays bound even in the absence of the transferable acetyl group, we conclude that the cleavage products stay associated with the KAT domain until the next round of modification is initiated.

Structural comparison between bacterial and archaeal Elp3. DmcElp3 represents a rare example of bacterial Elp3s, but shares high sequence similarity with archaeal Elp3s, which also lack genes for all the other five eukaryotic Elongator subunits. Although DmcElp3 and Methanocaldococcus infernus Elp3 (MinElp3) share 50\% sequence identity, MinElp3 harbors an extended N-terminus (aa 1-75). This flexible region enriched in basic residues is present in most archaeal and eukaryotic Elp3 sequences, but completely absent from DmcElp3 ${ }^{21}$. To gain further structural and functional insights into archaeal and more closely related eukaryotic Elp3s, we expressed and purified recombinant full length MinElp3 under aerobic conditions. Unlike DmcElp3, which shows two discrete oligomeric states ${ }^{21}$, full length MinElp3 exclusively forms monomers. We further generated five different N-terminally truncated versions $\left(\right.$ MinElp $_{\Delta 1-19}, \quad$ MinElp3 $3_{\Delta 1-41}, \quad$ MinElp $3_{\Delta 1-46}, \quad$ MinElp $3_{\Delta 1-54}$, MinElp3 $\left.3_{\Delta 1-68}\right)$ to facilitate crystallization. In comparison to the full length protein, all five truncations exhibited two discrete concentration-independent oligomeric states in size exclusion 
Table 1 Data collection, phasing and refinement statistics

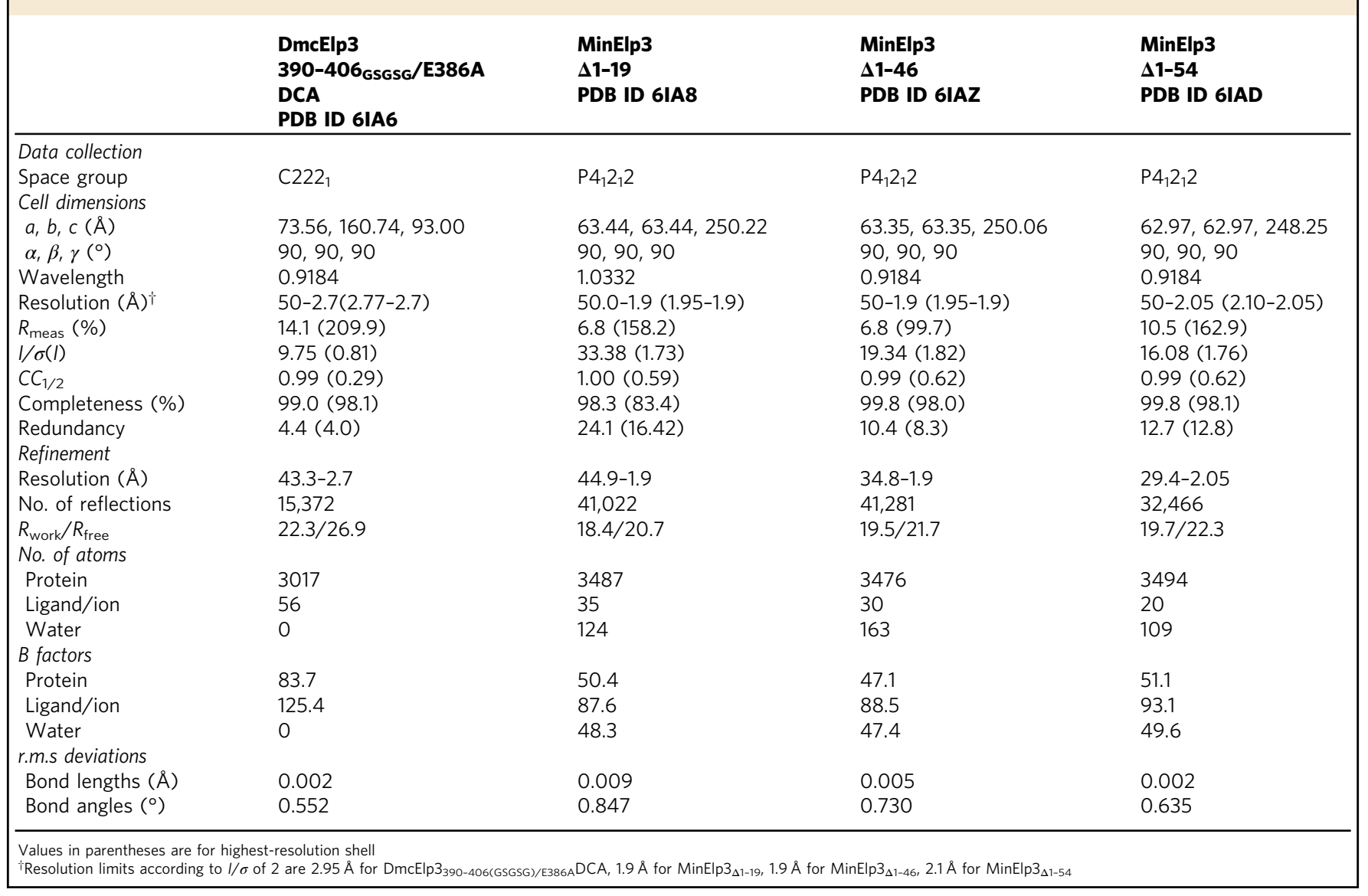

chromatography, namely monomers and dimers (Supplementary Fig. 2a). All purified MinElp3 samples appeared slightly pink, suggesting the presence of an $\mathrm{Fe}-\mathrm{S}$ cluster in a minority of monomeric and dimeric MinElp3 molecules even under aerobic purification conditions. Moreover, the $\mathrm{Fe}-\mathrm{S}$ cluster loop (like in DmcElp3) directly contributes to the dimer formation of truncated MinElp3 (Supplementary Fig. 2a). Notably, the monomeric forms of truncated MinElp3 elute earlier from the gel filtration column and therefore appear to be larger than expected. We confirmed their theoretical masses by SDS-PAGE and light scattering (RALS and LALS) measurements and speculate that the $\mathrm{N}$-terminus could also be involved in the compaction of the bacterial and archaeal Elp3 proteins.

We used full length and truncated versions of purified MinElp3 in crystallization trials and obtained well-diffracting crystals for monomeric MinElp3 $3_{\Delta 1-19}$, MinElp3 $3_{\Delta 1-46}$, and MinElp3 $3_{\Delta 1-54}$. We solved their structures using a poly-alanine model of DmcElp3 via molecular replacement at $1.9 \AA, 1.9 \AA$, and $2.1 \AA$ resolution, respectively (Fig. $2 \mathrm{a}$ and Table 1 ). Although all three versions contained various lengths of the $\mathrm{N}$-terminus and also showed no signs of degradation, we could observe interpretable electron density only starting from residue V72 in all collected and refined datasets. Therefore, we believe that the $\mathrm{N}$-terminus adopts a highly flexible conformation in the absence of substrate tRNAs. The overall structure and domain arrangement and interface between the rSAM and KAT domains of MinElp3 are almost identical to DmcElp3 (Supplementary Fig. 2b,c). The main difference relates to the Fe-S cluster loop region (aa 87-123) and the $\mathrm{Fe}-\mathrm{S}$ cluster itself, which were both completely unstructured in all monomeric MinElp3s (Fig. 2a and Supplementary Fig. 2d). We previously identified a Zn-binding motif in the central linker region between the two domains that is coordinated by three cysteine residues, which are strictly conserved in bacterial and archaeal but missing in eukaryotic Elp3s (Fig. 2b) ${ }^{21}$. In our MinElp3 structures, we could not locate any bound $\mathrm{Zn}$-ions and instead observed the formation of a disulfide bond between C384 and C389. Nevertheless, the equivalent sequence still obtains an almost identical conformation, indicating that zinc might simply contribute structural integrity in anaerobic bacteria. In addition, we observed that the acetyl-CoA blocking loop (aa 466-486) is positioned outside of the acetyl-CoA binding pocket, interacting with the last visible residues of the $\mathrm{N}$-terminus and also contacting a neighboring symmetry mate (Fig. 2c and Supplementary Fig. 2e). Using our DmcElp3-DCA structure as a reference model, we are able to show that acetyl-CoA would also fit seamlessly into the active site of MinElp3, indicating an almost identical ligand coordination and active site organization. In summary, bacterial and archaeal Elp3 proteins do not only share high sequence conservation (Supplementary Fig. 2d and Supplementary Fig. 3a), but also adopt an almost identical overall domain arrangement indicating that they represent examples of the genuine structure of all Elp3 proteins.

Functional comparison between bacterial and archaeal Elp3. It was recently shown that the acetyl-CoA hydrolysis activity of MinElp3 can be induced by the presence of in vitro transcribed and truncated tRNAs and that this trigger does not require the presence of an $\mathrm{Fe}-\mathrm{S}$ cluster ${ }^{20}$. We compared the acetyl-CoA hydrolysis rates of DmcElp3 and MinElp3 using purified endogenous bulk tRNAs from an Elp3-depleted yeast strain, which carries naturally occurring modifications. Both proteins exhibited low intrinsic basal hydrolysis rates in the absence 
a
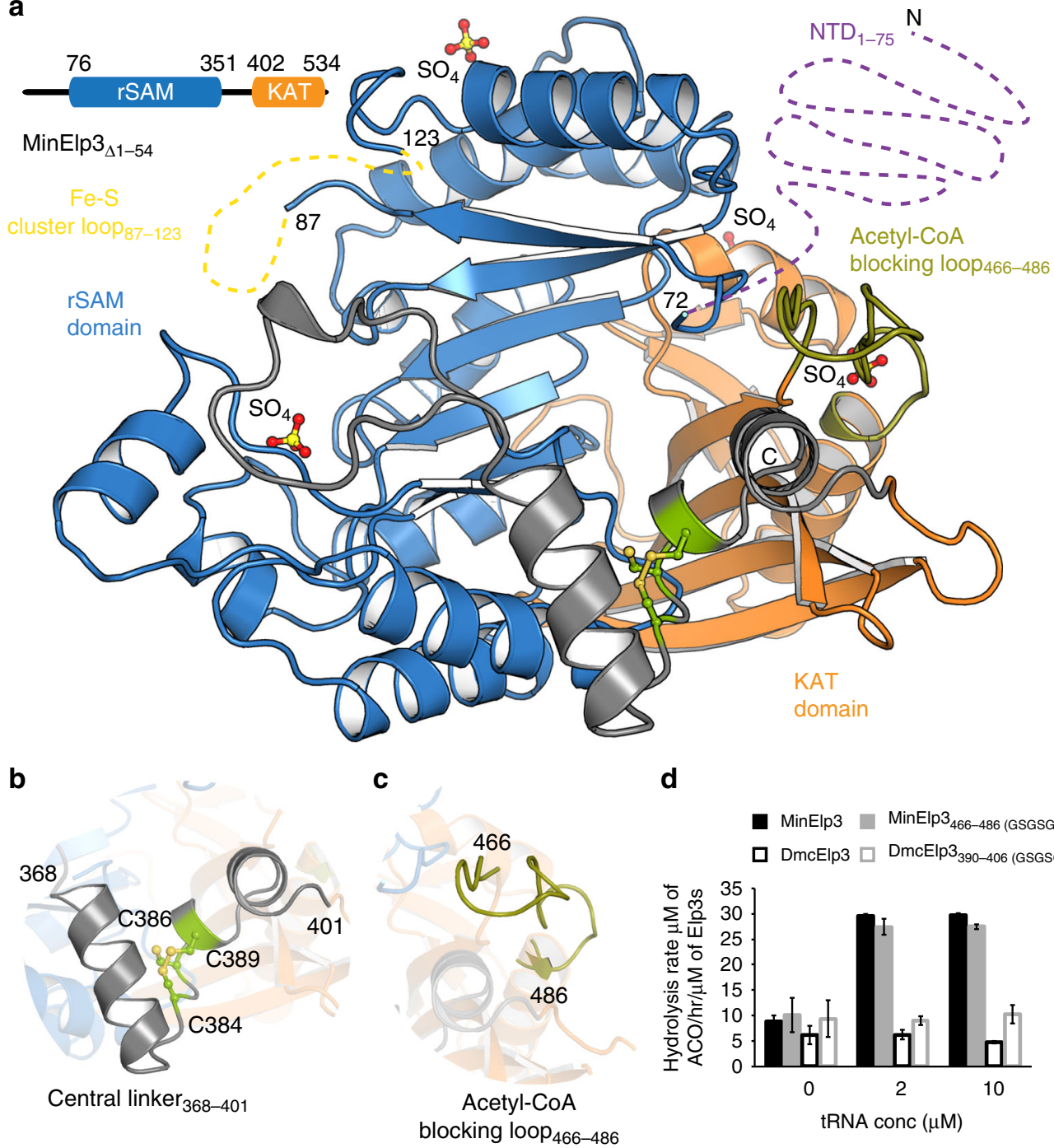

Fig. 2 The crystal structure of MinElp3 $3_{\Delta 1-54}$ a Overall structure of MinElp3 ${ }_{\Delta 1-54}$ in cartoon representation. The rSAM domain (blue), KAT domain (orange), acetyl-CoA blocking loop (olive), and central linker region (gray) are highlighted. Disordered loops are shown using dotted lines. Cysteines in the central linker region are highlighted in green and $\mathrm{SO}_{4}$ is shown as orange and red spheres. The respective amino acid numbers are indicated. $\mathbf{b} \mathrm{Close-up} \mathrm{view} \mathrm{of}$ the central linker region that connects rSAM and KAT domains. The three cysteine residues involved in disulfide bond formation are indicated (yellow). $\mathbf{c}$ Close-up view of the acetyl-CoA blocking loop (olive). d The acetyl-CoA hydrolysis rates of MinElp3, MinElp3466-486(GSGSG), DmcElp3 and DmcElp3 $390-406$ (GSGSG) in the absence and presence of endogenous yeast bulk tRNA ( $\triangle$ elp3; UMY2916). Data represent mean \pm standard error of the mean (SEM). Source data are provided as a Source Data file

of tRNA, but MinElp3 as well as its mutated form lacking the acetyl-CoA blocking loop (MinElp3 $3_{466-486(G S G S G)}$ ) showed robust

acetyl-CoA hydrolysis activity after addition of bulk tRNA. Furthermore, we confirmed that MinElp3 binds to acetyl-CoA $\left(K_{\mathrm{d}}\right.$ $135.4 \pm 55.3 \mu \mathrm{M})$ and that deleting the acetyl-CoA blocking loop does not have significant effects on its affinity to acetyl-CoA in the absence of tRNA $\left(K_{\mathrm{d}} 107.7 \pm 55.8 \mu \mathrm{M}\right.$; Supplementary Fig. 3b). DmcElp3, on the other hand, showed $\sim 5$-times reducedinduced hydrolysis activity, which remained similarly weak after deletion of the acetyl-CoA blocking loop region (DmcElp3 390-406(GSGSG); Fig. 2d). The varying levels of inducibility correlate well with differences in the binding affinities of MinElp3 $\left(K_{\mathrm{d}} 50 \pm 10 \mathrm{nM}\right)$ and DmcElp3 $\left(K_{\mathrm{d}} \sim 1410 \pm 230 \mathrm{nM}\right)$ towards Cy5-labeled in vitro transcribed tRNA(Dmc)tRNAGlu $_{\text {UUC }}$ (CCA) (Supplementary Fig. 3c, d). As described above, the flexible $\mathrm{N}$-terminus shows the biggest sequence variation among all known Elp3 proteins and also represents the main difference between MinElp3 and DmcElp3 sequences (Fig. 3a). Therefore, we analyzed if the extended flexible $\mathrm{N}$-terminus of MinElp3 could account for the differences in tRNA binding affinity using electromobility shift assay (EMSA) and microscale thermophoresis (MST). We observed strong interaction of the purified MinElp3 N-terminus (MinElp3 $3_{1-77}$ ) towards (Dmc)tRNA Glu $_{U U C}$ and confirmed decreased affinities for N-terminally truncated MinElp3 proteins (Fig. 3b). We have designed and purified truncated versions sampling the whole $\mathrm{N}$-terminus based on secondary structure predictions with the aim of conserving potential helices and referring stability (Fig. 3a). The first 45 residues seem to have a particularly strong contribution to the binding and deletion of the complete $\mathrm{N}$-terminus resulted in affinities comparable to DmcElp3 (Fig. 3c). The decrease in tRNA affinities correlates well with a strong decrease in tRNA-induced acetyl-CoA hydrolysis activity of MinElp3, thereby confirming the specificity of the induction and emphasizing the contribution of Elp3 $\mathrm{N}$-termini to tRNA binding and modification activity (Fig. 3d). As the 
a

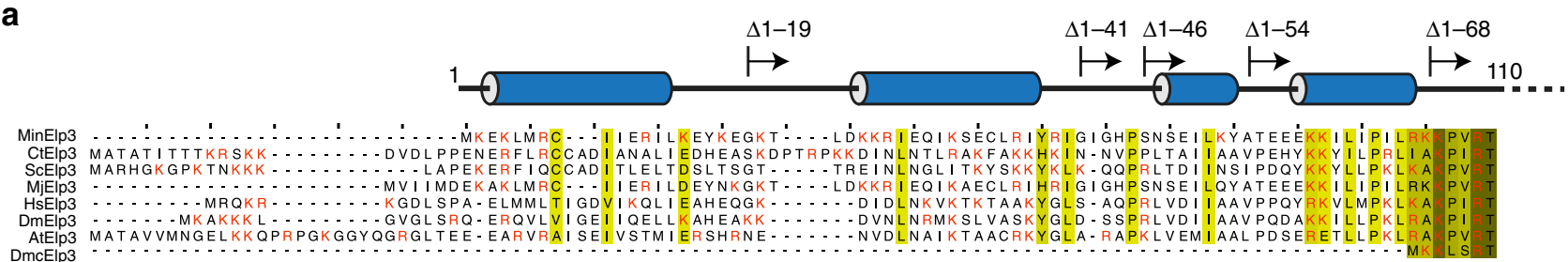

b

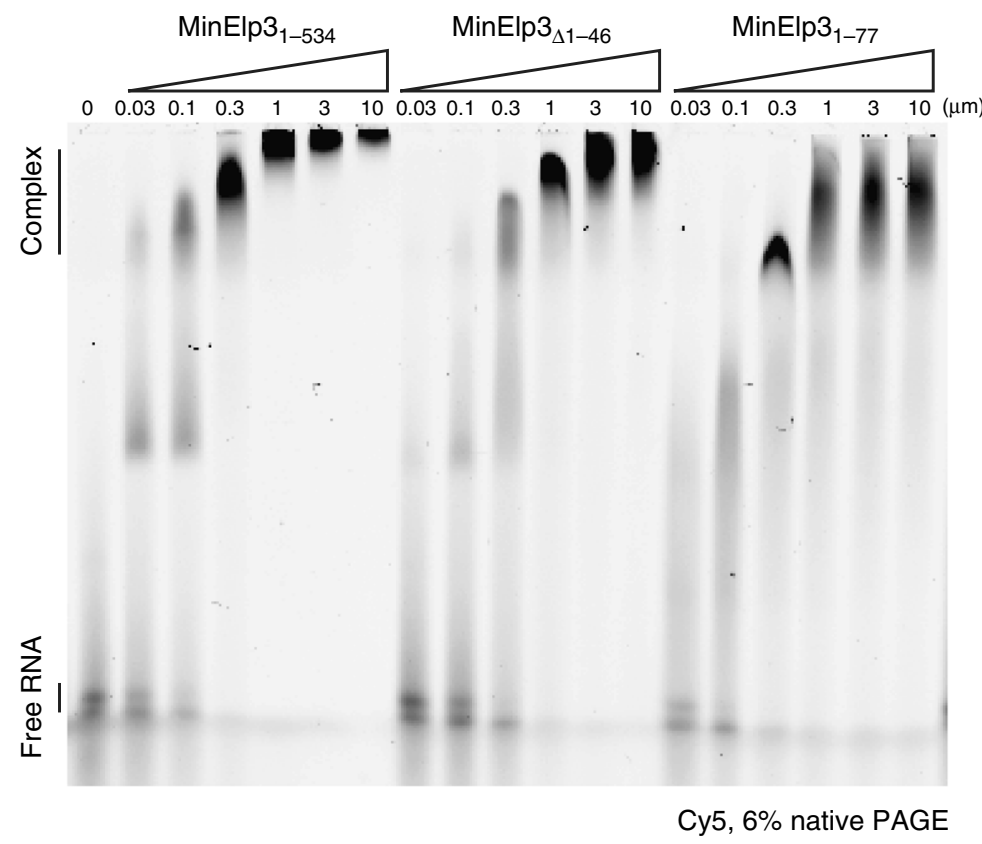

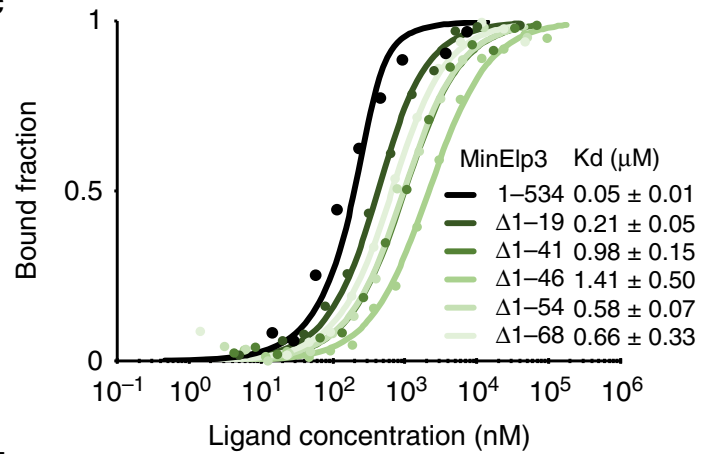

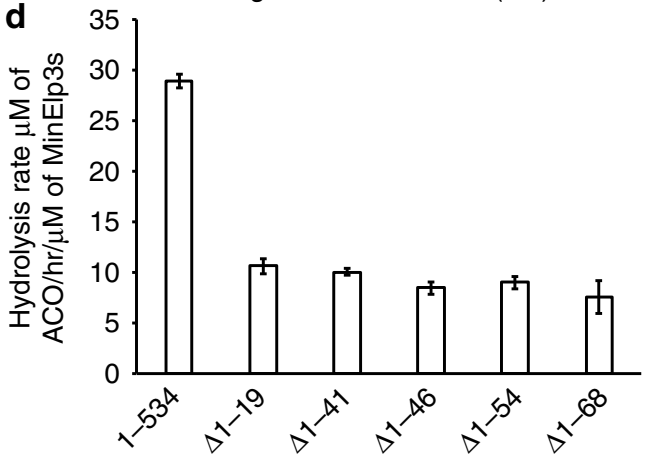

Fig. $3 \mathrm{~N}$-terminus features of MinElp3 in tRNA binding and acetyl-CoA hydrolysis. a Sequence alignment of N-termini in selected Elp3 proteins. D. mccartyi (DmcElp3) (YP307690), M. infernus (MinElp3) (YP003616086), M. jannaschii (MjElp3) (AAB99138), S. cerevisiae (ScElp3) (NP015239), D. melanogaster (DmElp3) (NP608834), A. thaliana (AtElp3) (NP568725), H. sapiens (HsElp3) (NP060561), C. thermophilum (CtElp3) (XP_006697164.1). Relatively conserved residues are labeled in gradient green depending on the conservation scores. Basic residues are labeled in red. The predicted secondary structure elements of MinElp3 are indicated on top of the aligned sequences. The individual start sites of the truncated N-terminal constructs are indicated. b EMSA analyses of MinElp3s binding to yeast tRNA Ser $_{\text {UGA }}$ (Cy5-labeled). The samples were resolved in a $6 \%$ native PAGE and visualized using a Typhoon scanner. Protein concentrations are given and positions of free tRNA and protein-tRNA complex are labeled. c MST analyses of MinElp3s binding to (Dmc) tRNA ${ }^{\text {Glu }}$ uuc and the $K_{\mathrm{d}} \mathrm{s}$ are indicated, $n=3$. d Acetyl-CoA hydrolysis reaction of different MinElp3 N-terminus truncations in the presence of endogenous yeast bulk tRNA ( $\Delta$ elp3; UMY2916), $n=3$. Data represent mean \pm SEM. Source data are provided as a Source Data file

$\mathrm{N}$-terminal regions of Elp3 proteins are evolutionary very weakly conserved, we were curious if exchanging these sequences between species would affect the functionality. Strikingly, replacing the N-terminus of MinElp3 (aa 1-69) with the respective yeast (aa 1-95) or human (aa 1-78) sequences restored the tRNA binding affinity of N-terminally truncated MinElp3 (Supplementary Fig. 3d). As the tRNA-induced acetyl-CoA hydrolysis rates remained unchanged in these chimeric proteins (Supplementary Fig. 3e), it seems that although tRNA recognition is conserved, species-specific mechanisms is required to trigger the KAT activity.

The acetyl-CoA hydrolysis of Elp3 is tRNA dependent. Next, we tested whether other nucleic acids or the absence of charged amino acids could play a role for Elp3 activation. Both, bulk tRNA from wild yeast strains carrying endogenous levels of Elongator modifications, as well as de-acylated bulk tRNAs, efficiently activated acetyl-CoA hydrolysis of MinElp3 (Fig. 4a and Supplementary Fig. 4a, b). In contrast, neither polyU RNA nor single stranded DNA showed any detectable induction of hydrolysis rates. It has been an ongoing debate for many years, whether Elp3 could also catalyze the canonical acetylation of lysine residues in peptides and proteins. Therefore, we synthesized and tested lysine-containing histone ( $\mathrm{H}_{10-18}$ and $\left.\mathrm{H}_{4-12}\right)$ and tubulin (Tubulin T6-44 ) peptides, which have previously been suggested $^{18,51}$ as potential Elongator substrates and shown to be suitable GCN5 substrates ${ }^{52-54}$ (Fig. 4b). Strikingly, neither histone nor tubulin peptides are able to bind archaeal MinElp3 (Supplementary Fig. 4c) nor trigger its acetyl-CoA hydrolysis reaction (Fig. 4a). Although in our hands all tested eukaryotic Elp3 proteins are highly unstable and insoluble in the absence of Elp1 and Elp2, we managed to obtain pure and homogenous KAT domain from Chaetomium thermophilum (CtElp3 ${ }_{435-574}$ ), a thermophilic yeast strain ${ }^{55}$. All employed peptides failed to induce the enzymatic activity of this isolated KAT domain from this eukaryotic Elp3, even though the predicted peptide-binding site is principally exposed due to the absence of the rSAM domain (Supplementary Fig. 4d, e). Elp3 requires both domains to bind tRNAs to activate acetyl-CoA hydrolysis, explaining the observation that bulk tRNA also failed to induce the enzymatic activity of this eukaryotic Elp3 KAT domain. 
a

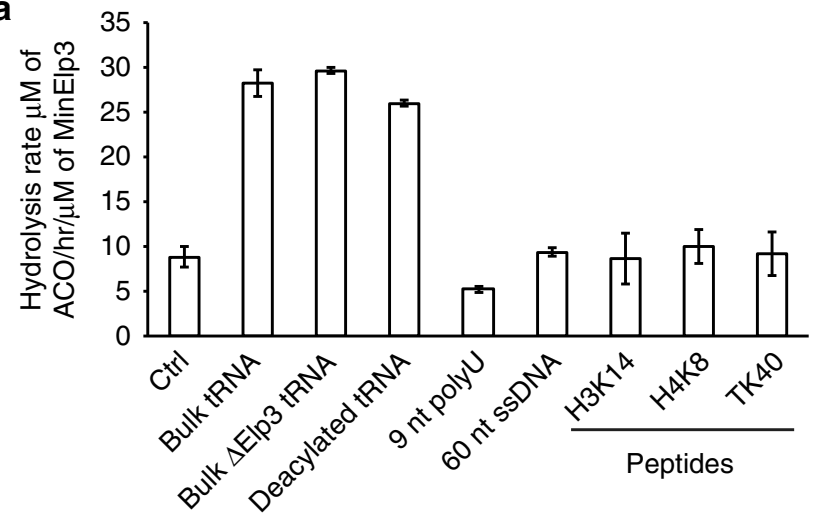

b
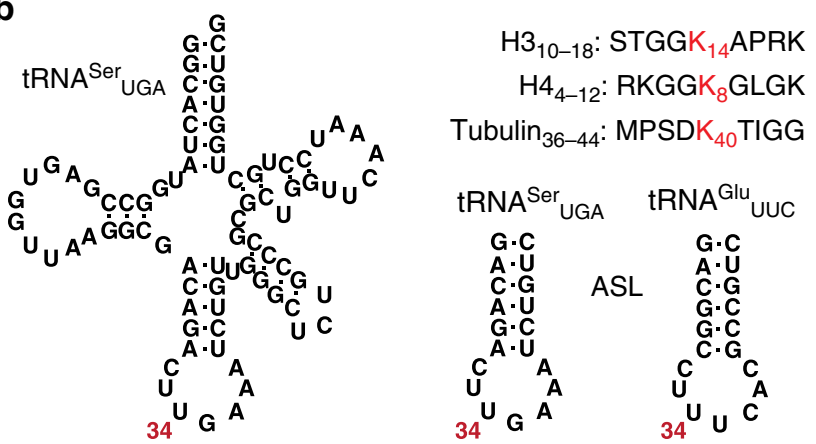

c

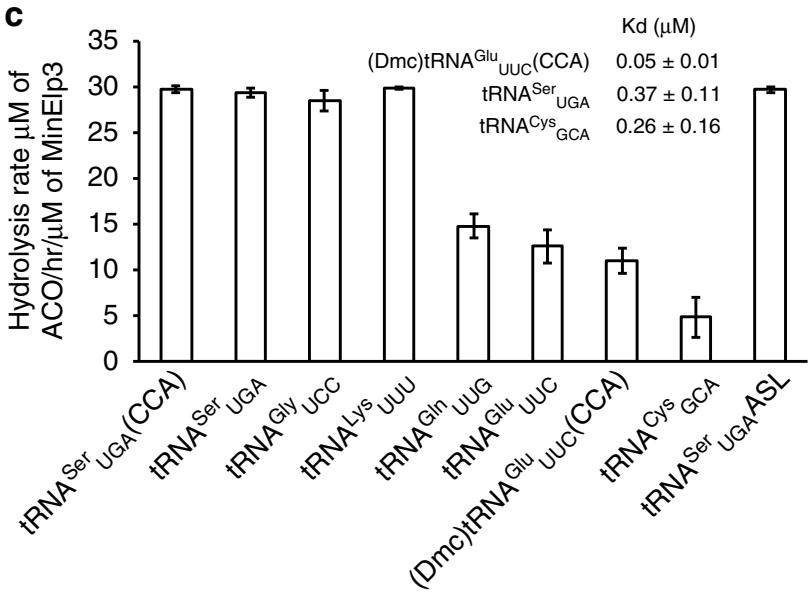

d

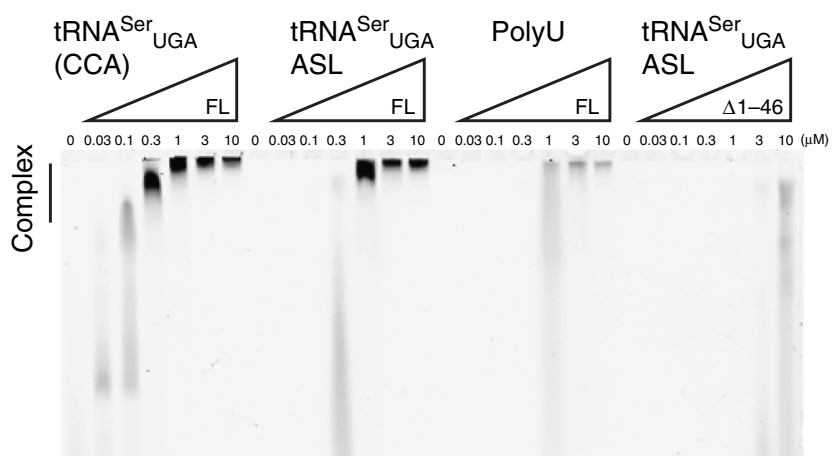

d

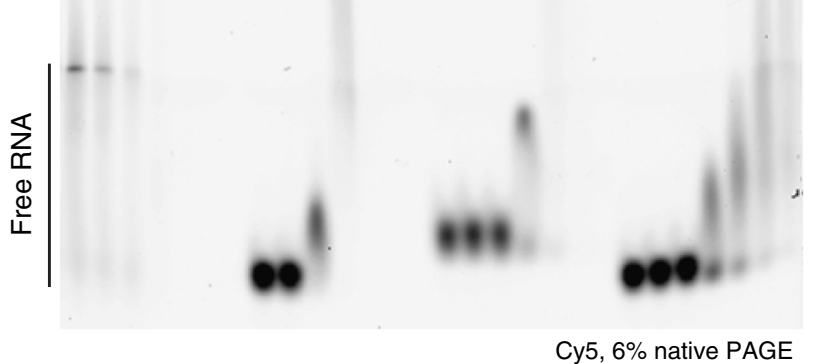

Cy5, 6\% native PAGE

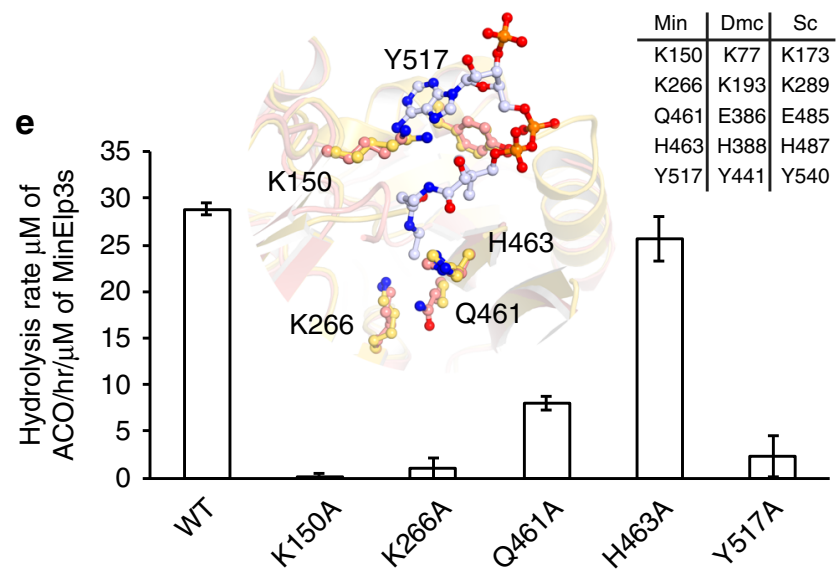

Fig. 4 Substrate specificity feature of MinElp3 to activate acetyl-CoA hydrolysis. a Acetyl-CoA hydrolysis reactions of MinElp3 in the presence of different substrates, $n=3$. $\mathbf{b}$ Chemical structure illustrations of tRNA and ASLs. Sequences of used peptides are given and modifiable lysine (K) residues are highlighted. $\mathbf{c}$ Acetyl-CoA hydrolysis reactions of MinElp3 in the presence of different tRNA substrates, $n=3 \mathbf{d}$ EMSA analyses of MinElp3s binding to different nucleic acid substrates. Protein concentrations are given and positions of free tRNA and protein-tRNA complex are labeled. e Acetyl-CoA hydrolysis reactions of different MinElp3 point mutations in the presence of endogenous yeast bulk tRNA ( $\Delta$ elp3; UMY2916), $n=3$. Superposition of highlighted residues in the acetyl-CoA binding pocket from MinElp3 (light pink) and DmcElp3 (yellow). DCA is shown in ball and sticks representation. The equivalent residue numbers in different Elp3s are indicated. Data represent mean \pm SEM. Source data are provided as a Source Data file

The tRNA substrate selectivity of Elp3. We subsequently analyzed whether any tRNA might trigger the acetyl-CoA hydrolysis of Elp3 or if the protein is able to select for suitable tRNA substrates. We initially noticed no binding preference for different in vitro transcribed tRNAs as the affinities for tRNA ${ }^{\text {Cys }}{ }_{\mathrm{GCA}}$, tRNA $^{\text {Ser }}{ }_{U G A}$, and tRNA ${ }^{\text {Glu }}{ }_{U U C}$ are in a comparable low nanomolar affinity range (Fig. $4 \mathrm{c}$ and Supplementary Fig. 5a). Therefore, MinElp3 binds equally well to unmodifiable (tRNA${ }_{\text {Cys }}{ }_{\text {GCA }}$ ) and modifiable (tRNA ${ }^{\text {Ser }}{ }_{\text {GAA }} /$ tRNA $^{\text {Glu }}{ }_{U U C}$ ) tRNAs and is not capable of specifically recognizing the respective RNA base present at position 34 . We subsequently tested several in vitro transcribed tRNAs, including tRNA ${ }^{\mathrm{Lys}}{ }_{\mathrm{UUU}}, \mathrm{tRNA}^{\mathrm{Ser}}{ }_{\mathrm{UGA}}$, tRNAGly $_{\text {UCC }}$, and tRNA ${ }^{\text {Cys }}{ }_{\text {GCA }}$, for their potential to induce acetyl-CoA hydrolysis. Whereas, tRNA ${ }^{\mathrm{Ser}_{\mathrm{UGA}}}$ and tRNA ${ }^{\mathrm{Gly}}{ }_{\mathrm{UCC}}$ triggered the hydrolysis as well as endogenous bulk tRNA, we did not observe induction using tRNA ${ }^{\mathrm{Cys}}{ }_{\mathrm{GCA}}$, which harbors $\mathrm{G}_{34}$ (Fig. 4c). It appears that Elp3 shows broad specificity for tRNA binding, but strictly discriminates for modifiable $U_{34}$-tRNAs at the step of acetyl-CoA hydrolysis. In addition, Elp3 seems to work independently from priming modification pathways, as endogenous fully decorated and in vitro transcribed "naked" tRNAs show an identical potential to induce its activity. 
a
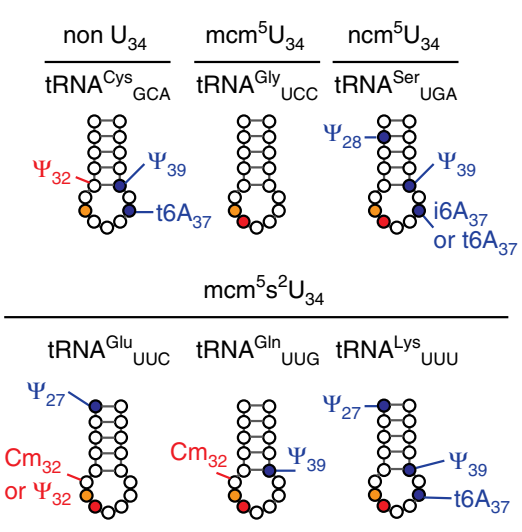

b
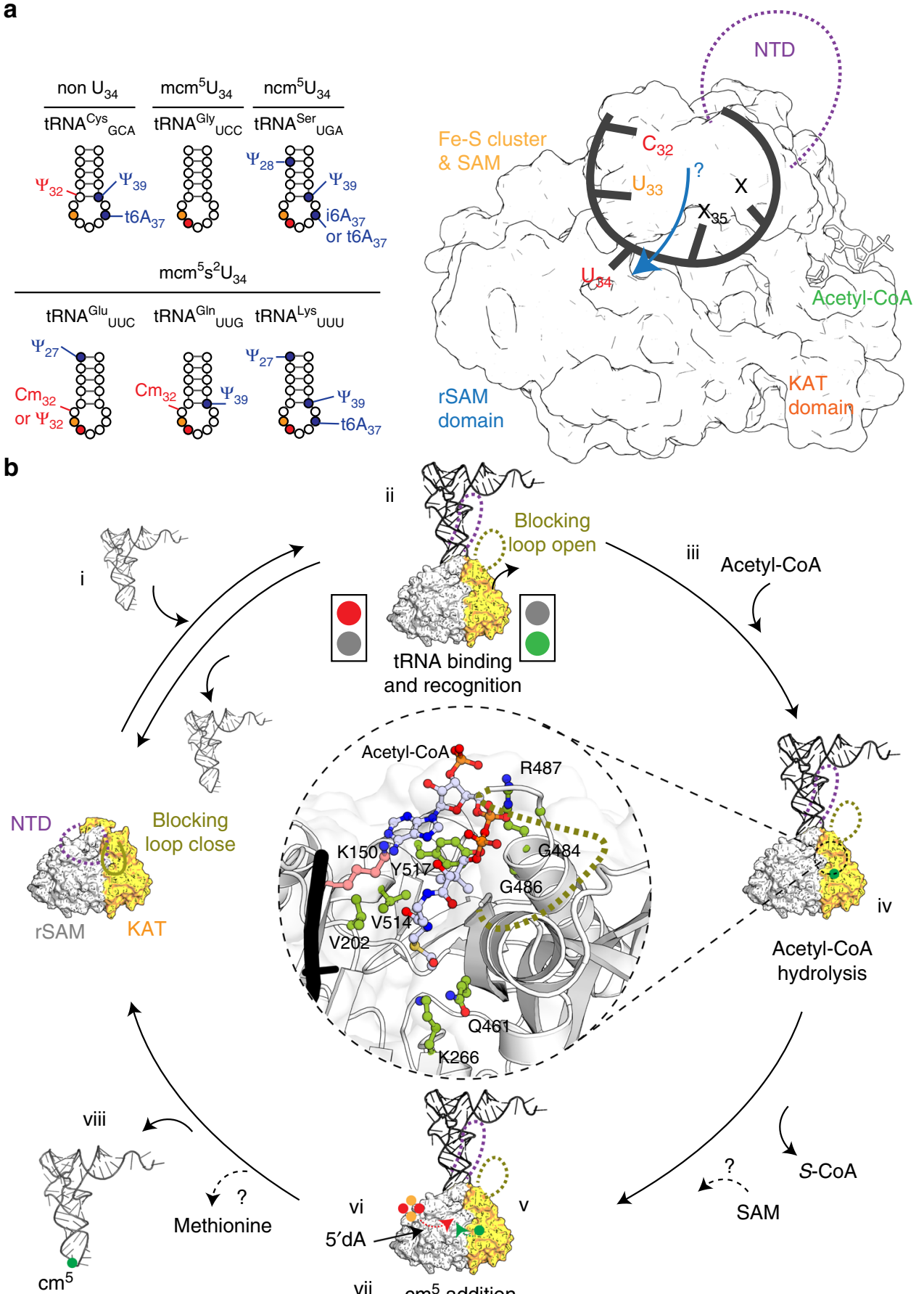

ii
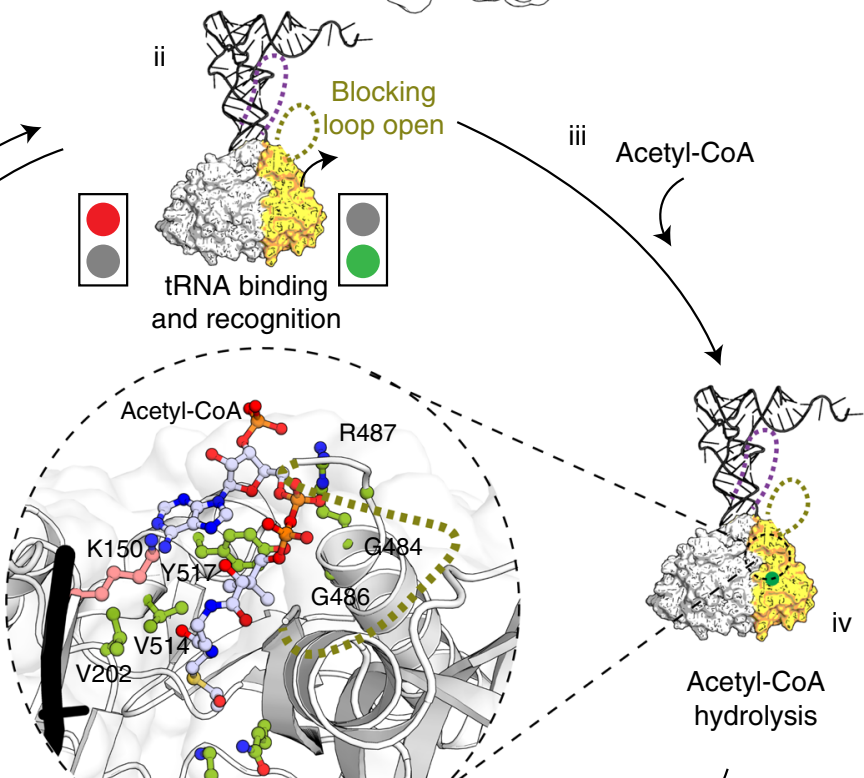

Fig. 5 Proposed structural rearrangements of Elp3 during tRNA modification. a (left) Experimentally proven modifications in ASLs of different types of tRNAs. $U_{34}$ is shown as red circles and the invariable $U_{33}$ is shown as orange circles. Most common modifications are indicated (blue) while the

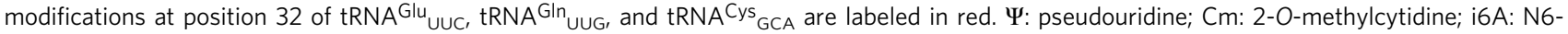
isopentenyladenosine; t6A: N6-threonylcarbamoyladenosine. (right) The proposed influence of priming modification on the $\mathrm{cm}^{5}$ modification mechanism at $U_{34}$ (blue arrow). b Schematic overview of the individual sequential steps in the Elp3-mediated tRNA modification cycle. The $\mathrm{N}$-terminus could change its location upon tRNA binding that regulates the position of acetyl-CoA blocking loop. The acetyl-CoA hydrolysis responsible residues (pink) and acetylCoA binding residues (green) are indicated in the inset. SAM is required for the catalytic reaction; however, the timing of SAM recruitment and release of the methionine by-product are still not clear (indicated by question marks)

Next, we analyzed whether Elp3 discriminates between different modifiable tRNAs as $\mathrm{cm}^{5} \mathrm{U}_{34}$ primes for three downstream cascades, namely $\mathrm{mcm}^{5} \mathrm{U}_{34^{-}}, \mathrm{ncm}^{5} \mathrm{U}_{34^{-}}$and $\mathrm{mcm}^{5} \mathrm{~s}^{2} \mathrm{U}_{34}$. Therefore, we tested tRNA ${ }^{\mathrm{Glu}}{ }_{U U C}, \mathrm{tRNA}^{\mathrm{Gln}}{ }_{U U_{G}}$ and tRNA ${ }^{\mathrm{Lys}} \mathrm{UUU}$ all harboring $\mathrm{mcm}^{5} \mathrm{~s}^{2} \mathrm{U}_{34}$ and an invariant $\mathrm{U}_{35}$ on their ability to induce acetyl-CoA activity. Strikingly, we observed regular levels of activity for tRNA ${ }^{\mathrm{Lys}} \mathrm{UUU}$, but only intermediate activities for tRNA Glu $U$ UUC, tRNA ${ }_{\text {UUG }}$ and (Dmc)tRNA Glu ${ }_{U U C}$ (Fig. 4c). Initially, we were curious, if $\mathrm{U}_{35}$ next to $\mathrm{U}_{34}$ could cause a decreased acetyl-CoA hydrolysis activity, which is somehow restored in for tRNA ${ }^{\mathrm{Lys}} \mathrm{UuU}$. As the substitution of $\mathrm{U}_{35}$ to either purine $\left(\mathrm{G}_{35}\right)$ or pyrimidine $\left(\mathrm{C}_{35}\right)$ in yeast tRNA ${ }^{\text {Glu }}$ did not elevate 
its ability to induce the activity of MinElp3 (Supplementary Fig. 5b), we conclude that other determinants in tRNA ${ }^{\text {Glu }}{ }_{U U C}$ and tRNA ${ }^{\text {Gln }}$ UUG must be causative for their decreased activity.

We previously have shown that DmcElp3 not only uses its central basic cleft to bind the ASL of a tRNA, but also employs other basic residues residing in the $\mathrm{N}$-terminus to make additional contacts around the D-loop of tRNAs ${ }^{21}$. To investigate whether these additional contacts are required for the enzymatic activation in MinElp3, we tested whether the ASL (derived from tRNA $^{\text {Ser }}{ }_{\text {UAA }}$ ) is sufficient to activate Elp3. We observed that the ASL by itself is not only sufficient to bind to full length MinElp3, but also induces enzymatic activity at a comparable level as full length tRNA $^{\mathrm{Ser}_{U G A}}$ (Fig. $4 \mathrm{c}, \mathrm{d}$ ). These observations are in line with a report that MinElp3 can modify tRNA ${ }^{\text {Arg }}$ that misses Dand T-loop ${ }^{20}$. We have previously reported that the ASL of (Dmc)tRNA ${ }^{\text {Glu }}{ }_{U U C}$ is not sufficient to bind to bacterial DmcElp3. In agreement with this data and the described importance of the extended N-terminus in MinElp3, we observed that the central basic cleft of MinElp3 (Supplementary Fig. 5c) is not sufficient for efficient ASL binding. The affinity between the truncated MinElp $3_{\Delta 1-46}$ protein and the ASL alone are comparable to the one of an unspecific polyU sequence towards full length MinElp3 (Fig. 4d). As the presence of a 3'CCA sequence, added during the maturation of tRNAs, increases the affinity of full length MinElp3 for different tRNAs (Supplementary Fig. 5a), we speculate that the extended N-terminus might be involved in both, the recognition of the ASL and the 3'CCA regions.

Identification of active site residues in the KAT domain. To identify the functional role of individual amino acids in the acetyl-CoA binding pocket of Elp3, we introduced several structure-guided mutations in MinElp3 (K150A, K266A, Q461A, $\mathrm{H} 463 \mathrm{~A}$, and Y517A) and tested them for acetyl-CoA hydrolysis activity (Fig. 4e). Among these variants, only H463A still showed acetyl-CoA hydrolysis activity, whereas all remaining MinElp3 mutations (K150A, K266A, Q461A, and Y517A) showed strongly reduced or completely diminished tRNA-induced acetyl-CoA hydrolysis activity. The reduced hydrolysis activity of the affected mutants is not caused by alteration in their tRNA binding (Supplementary Fig. 6a), but reduced acetyl-CoA binding for K266A, Q461A, and Y517A. As K150A shows normal tRNA and acetyl-CoA binding, we believe that it might be directly involved the hydrolysis reaction (Supplementary Fig. 6b). Our previous in vivo analyses of the equivalent positions in yeast Elp3 (ScElp3) based on the apo structure of DmcElp3 confirmed the functional relevance of these residues for the eukaryotic Elongator complex $^{21}$. To provide a complete mechanistic picture of the active site, we have undertaken major efforts to co-crystalize different Elp3 proteins in complex with various tRNA sequences, but to date have not succeeded in obtaining analyzable crystals. Future structural studies will take advantage of the identified mutants and use different combinations of ASLs and acetyl-CoA derivatives to lock Elp3 in different reaction intermediates.

\section{Discussion}

Catalytic reactions and regulatory mechanisms of tRNA modification enzymes are diverse and complex ${ }^{7}$. The enzymatically active Elp3 subunit of Elongator contains two functional domains, utilizes multiple cofactors, and conducts a unique uridine base modification specifically found only at the wobble base position of tRNAs. Although, all known Elp3s share high sequence similarity, eukaryotic Elp3s are embedded in the large Elongator complex and the modification reaction requires five other Elongator subunits and several regulatory factors ${ }^{56,57}$. Nevertheless, it was recently shown that $(\mathrm{m}) \mathrm{cm}^{5} \mathrm{U}_{34}$ is present in
tRNAs from $H$. volcanii indicating that archaeal Elp3s must be able to conduct a very similar modification reaction as the fully assembled eukaryotic Elongator complex ${ }^{58}$. In this study, we provide a comprehensive structural and functional comparison of bacterial, archaeal and eukaryotic Elp3 proteins and their KAT domains.

Our results clearly illustrate that Elp3 can recognize and respond to in vitro transcribed tRNA substrates as well as to endogenous bulk tRNAs from yeast. We also demonstrate that a synthesized ASL can induce enzyme activity, whereas other tRNA sequences (e.g. tRNA ${ }^{\mathrm{Glu}} \mathrm{UUC}_{\mathrm{C}}, \mathrm{tRNA}^{\mathrm{Gln}} \mathrm{UUG}_{\mathrm{UG}},(\mathrm{Dmc}) \mathrm{tRNA}^{\mathrm{Glu}} \mathrm{UUC}$, and tRNA $\left.{ }^{\mathrm{Cys}}{ }_{\mathrm{GCA}}\right)$ show reduced or no activity at all. These data indicate that specific bases in the ASL of tRNA are necessary to activate Elp3 and that for certain modifiable tRNAs additional modification might also be necessary for full activation (Supplementary Fig. 7). Thus, we searched the MODOMICS database ${ }^{1}$ of experimentally proven tRNA modifications in eukaryotic organisms and found that there are two specific modifications in the ASL of tRNA ${ }^{\text {Glu }}{ }_{U C}$ and tRNA ${ }^{\text {Gln }}{ }_{U U G}$, which are not found in tRNA $_{\text {Lys }}^{\text {Ly }}$ or other modifiable tRNAs (Fig. 5a). They carry a pyrimidine modification at position 32 (either $\mathrm{Cm}_{32}$ or pseudouridine $\left(\Psi_{32}\right)$ ), which is absent in the used in vitro transcribed tRNAs. The lack of $\mathrm{Cm}_{32}$ is known to affect the anticodon stem loop structure and function ${ }^{7,59}$, and thereby could explain the reduced activity of Elp3 observed with these two tRNA species. Several studies have reported on the cross-talk and interdependency of tRNA modification networks ${ }^{60}$ and the link between these modifications and Elp3 activation needs to be investigated in the future.

By combining all available biochemical and structural data, we can conclude a potential mechanism of regulation and a sequential order of the Elp3 reaction cycle (Fig. 5b). (i) Modifiable and non-modifiable tRNAs bind to Elp3 with similar affinity; (ii) the N-terminus of Elp3 checks for the presence of 3'CCA and guides the ASL into a correct position within the central cleft of Elp3; (iii) tRNA binding displaces the acetyl-CoA blocking loop and could stimulate local structural rearrangement to facilitate acetyl-CoA binding, (iv) acetyl-CoA binding is secured and hydrolysis is initiated only by the correct modifiable substrate tRNAs in analogy to canonical acetyl transferases; (v) the released acetyl group is transported to the SAM domain; (vi) the Fe-S cluster generates a 5'dA-radical; (vii) an acetyl radical is generated via the contact of the acetyl group with the 5'dA-radical (viii) the acetyl radical is transferred onto $\mathrm{U}_{34}$ bound in close proximity and the modified tRNA leaves the complex.

Foremost, the presented crystal structures of DmcElp3-DCA and MinElp3 show striking similarities, indicating a common fold and domain architecture of all Elp3 family members. We confirmed the importance of several previously described active site residues for ligand binding and acetyl-CoA hydrolysis activity. Whereas, K266, Q461, and Y517 stabilize the binding of acetylCoA during the hydrolysis reaction, K150 might mimic a substrate lysine residue and trigger the acetyl transfer in the direction of the rSAM domain and the bound tRNA substrate. Our high resolution structural information of the unbound and ligandbound active site of the Elp3 KAT domain might facilitate the design and optimization of highly specific small compound inhibitors as research tools or for therapeutic applications.

In this respect, we established an acetyl-CoA hydrolysis assay, which allows us to quantitatively investigate the enzymatic activity of Elp3 as well as its substrate selectivity. Importantly, the assay format uncouples the KAT-based activity from the reductive SAM cleavage by the $\mathrm{Fe}-\mathrm{S}$ cluster in the rSAM domain, which would require fully anaerobic reconstitution of the involved $\mathrm{Fe}-\mathrm{S}$ cluster. We have employed this divide and conquer approach to identify the mechanism of substrate selection and 
specificity of Elp3 and Elongator. Although, we have repeatedly failed to reproduce a previously described modification assay that was based on isotope labelling ${ }^{20}$, we are able to show that archaeal Elp3 is able to discriminate between modifiable and unmodifiable tRNA substrates at the step of initiating acetyl-CoA hydrolysis.

Notably, the KAT domain of Elp3 responds specifically to tRNA molecules, whereas the addition of previously suspected peptide substrates or other types of nucleic acids does not trigger any detectable enzymatic activity. In particular, no stimulation of acetyl-CoA hydrolysis activity was observed in the case of an isolated eukaryotic Elp3 KAT domain. Although additional peptide binding sites on other eukaryotic Elongator subunits cannot be excluded, it appears, that the exposure of the hypothetical peptide binding site, which is normally blocked by the rSAM domain, does not stimulate a peptide-induced acetyl transfer reaction.

The recognition of substrate tRNAs by Elp3 is independent of other tRNA modifications, but for a subset of tRNA species priming ASL modification might increase the necessary ASL conformation and modification activity. The N-termini of Elp3 proteins seem to contribute to the specific recruitment of mature tRNAs carrying a 3'CCA, promote proper binding of the ASL into the active site and facilitate sequence-specific tRNA selection and specificity by sequence variation in different species. In summary, our data suggest that tRNAs represent the exclusive substrate for the eukaryotic Elongator complex as well as bacterial and archaeal Elp3 proteins, which all represent genuine tRNA editing enzymes.

\section{Methods}

Protein expression and purification. The MinElp3, CtElp3, ScElp3, and HsElp3 ORFs were synthesized and cloned into pETM30. The respective sequences are listed in Supplementary Table 1. Mutations were introduced using mutagenesis and truncations or N-termini of MinElp3 were made using PCR followed by restriction digestion and insertion of the amplified gene fragments into pETM30 or pETM11. The detailed sequences of the respective primers are listed in Supplementary Table 2 . The correct sequences of all constructs were confirmed by sequencing. All plasmids, including the DmcElp3 constructs ${ }^{21}$, were transformed into the strain BL21 (DE3) CodonPlus-RIL competent cells. Transformed cells were grown in TB broth and protein expressions were induced by isopropyl $\beta$-D-1-

thiogalactopyranoside IPTG $(1 \mathrm{mM})$ for overnight at $18^{\circ} \mathrm{C}$. The cells were collected and lysed in lysis buffer (50 mM HEPES, pH 7.5, $300 \mathrm{mM} \mathrm{NaCl}, 2 \mathrm{mM}$ DTT, $10 \%$ glycerol, $1 \mathrm{mM} \mathrm{MgCl}$, protease inhibitor, lysozyme and DNase). The spun supernatant was obtained by centrifugation at $80,000 \times g$ at $4{ }^{\circ} \mathrm{C}$ and subjected to a GSTPrep column for protein binding. The target protein was eluted by applying elution buffer containing $50 \mathrm{mM}$ HEPES, pH 7.5, $300 \mathrm{mM} \mathrm{NaCl}, 2 \mathrm{mM}$ DTT, $10 \%$ glycerol and $18 \mathrm{mM}$ GSH. The protein elute was collected and mixed with GSTTEV protease and dialyzed overnight to cleave off the GST tag. The protein solution was then applied back to a GSTPrep column to remove the GST-TEV protease and uncleaved protein. The flow-through was collected and applied to a heparin column and the protein was eluted by applying the gradient elution method (buffer condition: $20 \mathrm{mM}$ HEPES, pH 7.5, $3 \mathrm{M} \mathrm{KCl}, 2 \mathrm{mM}$ DTT). The protein elute was applied to a size-exclusion chromatography column (S200, 26/60, GE Healthcare) and the buffer was exchanged to $20 \mathrm{mM}$ HEPES, pH 7.5, $150 \mathrm{mM}$ $\mathrm{NaCl}, 5 \mathrm{mM}$ DTT. The protein concentration was measured and the concentrated purified protein was aliquoted and stored at $-80^{\circ} \mathrm{C}$ or subjected to crystallization trials. Protein mass of samples was determined using MALS/RALS (Malvern) equipped with a S200 gel filtration column (15/30, GE Healthcare). In the case of His-tagged MinElp3 $3_{1-77}$ protein, the purification step and conditions were the same as described above but using NiNTA beads for protein binding followed by 250 $\mathrm{mM}$ imidazole elution.

Protein crystallization and structure determination. Purified DmcElp3 $390-406$ (GSGSG)/E386A was concentrated to $10 \mathrm{~g} / \mathrm{L}$ and mixed with $1 \mathrm{mM}$ DCA (Jena Bioscience). The protein solution was combined with equal amount of crystallization buffer (100 mM MES, pH 6.3 and 4\% PEG 4000). The purified MinElp3 protein solutions $(12 \mathrm{mg} / \mathrm{ml})$ was prepared and crystallized at $22{ }^{\circ} \mathrm{C}$ in the condition of $0.1 \mathrm{M}$ phosphate/citrate (pH 3.8), $0.2 \mathrm{M} \mathrm{LiSO}_{4}$ and $12 \%$ PEG1000 or 0.1 $\mathrm{M}$ citrate (pH 3.8), $0.1 \mathrm{M} \mathrm{LiCl}$ and $17 \%$ PEG6000. All crystals were grown suing hanging-drop vapor-diffusion method. Crystals appeared after 3 days and were cryo-protected at the seventh day in a $30 \%$ glycerol solution and flash frozen in liquid nitrogen. Datasets were collected on a Pilatus $6 \mathrm{M}-\mathrm{F}$ detector at the BESSY II synchrotron (Berlin) for DmcElp3-DCA, MinElp3 $3_{\Delta 1-46}$, and MinElp3 $3_{\Delta 1-54}$, and DESY synchrotron (Hamburg) for MinElp3 $3_{\Delta 1-19}$. Data processing were performed with XDS/XSCALE ${ }^{61}$ and the structures were solved using Phaser ${ }^{62}$ and molecular replacement approach based on the existing DmcElp3 model (5L7L) and further refined using Phenix ${ }^{63}$. The refinement statistics of the respective models are given in Table 1 . The geometry and stereochemistry of the models were validated using MolProbity ${ }^{64}$. Structural models and superimpositions were analyzed and generated using $\mathrm{Pymol}^{65}$ and $\mathrm{Coot}^{66}$. For additional model validation of the presence of DCA, simulated annealing omit maps were calculated using phenix ${ }^{63,67}$. The electrostatic potential was calculated using $\mathrm{APBS}^{66}$ and PDB2PQR.

Peptide synthesis and purification. We synthesized three peptides (H3: YSTGGKAPRK; H4: YRKGGKGLGK; TK40: YMPSDKTIGG) that are known as acetyltransferase substrates ${ }^{18,50}$. We also include an additional tyrosine residue for facilitated concentration determination. All peptides were synthesized as Cterminal amides on a CEM Liberty Blue Microwave Peptide Synthesizer at 0.1 mmol scale by using standard Fmoc-solid phase peptide synthesis methodology on Rink Amide AM Resin $(0.9 \mathrm{mmol} / \mathrm{g}, 100-200 \mathrm{mesh})^{68}$. As recommended by manufacturer DIC/Oxyma Pure in DMF were used as coupling activators (in fivefold molar excess of Fmoc-AA-OH) and 10\% piperazine in NMP/ethanol (9:1) for deprotection. Following the final deprotection, N-terminal acetylation was performed with $10 \%$ acetic anhydride in DMF. Cleavage was performed for $3 \mathrm{~h}$ at room temperature with TFA/ $\mathrm{H}_{2} \mathrm{O} / \mathrm{TIS}(94 / 3 / 3)$. Following cleavage TFA solution was concentrated under nitrogen stream for $30 \mathrm{~min}$. The concentrated peptide solution was triturated with cold diethyl ether and the precipitate was centrifuged. Precipitated peptide was washed with diethyl ether and ethyl acetate (twice) and dried under vacuum overnight. Crude peptide was dissolved in $6 \mathrm{M}$ urea and purified by semipreparative RP-HPLC on C18 column. The purified fractions were collected, frozen, and lyophilized. Purified peptides were analyzed on analytical RPHPLC and confirmed by electrospray ionization mass spectrometry (ESI-MS). Peptides labeling with fluorescence probe was carried out after the solid phase peptide synthesis on the resin. In brief, dye conjugation was performed after coupling and deprotection of the last amino acid on the fully protected (except the $\mathrm{N}$-terminal amino group) peptide while still attached to the resin. Peptide resin (15 $\mu \mathrm{mols}$ ) slurry was prepared in $100 \mu \mathrm{l}$ anhydrous DMF (VWR Chemicals). Cyanine5 NHS ester (Abcam) was dissolved in anhydrous DMSO (Sigma) and it was added to peptide-resin slurry at equal molar ratio and the incubation was carried out at room temperature for $2 \mathrm{~h}$. i-Pr2NEt - N, N-Diisopropylethylamine (DIPEA, $50 \mathrm{mM}$ ) was then added dropwise to the slurry solution to adjust $\mathrm{pH}$ to 8.5 and followed by stirring overnight. The Cy5-peptide-resin was extensively washed with several solutions as follows: DMF, DCM, isopropanol, ethyl acetate, and dried under vacuum. Cleavage protocol and all post synthetic steps were the same as previously described procedure for non-labeled peptides. Cy5-labeled peptides were further purified using HPLC chromatography on the RP C18 column. Purified Cy5-peptides were lyophilized and stored at $-80^{\circ} \mathrm{C}$.

Yeast culture and bulk tRNA purification. The two $S$. cerevisiae strains (UMY2893 and UMY2916) ${ }^{69}$ were cultured from a single colony at $30^{\circ} \mathrm{C}$ for overnight with agitating at $200 \mathrm{rpm}$. The cells $\left(1 \mathrm{~L}, \mathrm{OD}_{600}\right.$ at 0.2$)$ were then further cultured until $\mathrm{OD}_{600}$ at 1.5 and followed by centrifugation at $3000 \times g$ at $4{ }^{\circ} \mathrm{C}$ for 10 min to collect cells. The cells were then washed with $\mathrm{H}_{2} \mathrm{O}$ and resuspended in $30 \mathrm{ml}$ TE buffer (10 mM Tris-HCl, pH 7.5, 1 mM EDTA) with addition of $0.1 \mathrm{M}$ $\mathrm{NaOAc}$ and $1 \%$ SDS. A cryo-mill operated with liquid nitrogen was used to grind and lyse cells. The powder was thawed on ice and followed by adding $30 \mathrm{ml}$ TRIzol (Thermo Fisher Scientific). The solution was vortexed for 1 min and incubated at RT for $20 \mathrm{~min}$. The RNA was purified by chloroform extraction and followed by two step precipitations in $8 \mathrm{M} \mathrm{LiCl}$ and followed by $0.3 \mathrm{M} \mathrm{NaOAc}$ and $75 \% \mathrm{EtOH}$ precipitation at $-20{ }^{\circ} \mathrm{C}$ for overnight. The RNA was spun into a pellet by centrifugation at $4000 \times g$ at $4{ }^{\circ} \mathrm{C}$ for $30 \mathrm{~min}$. The pellet was washed in $75 \% \mathrm{EtOH}$ for three times, air-dried and resuspended in $3 \mathrm{ml} 10 \mathrm{mM} \mathrm{NaOAc} \mathrm{pH}$ 5.2. The RNA was then further applied to a S200 gel filtration column (HiLoad 26/600) equilibrated in $10 \mathrm{mM} \mathrm{NaOAc}$ ( $\mathrm{pH}$ 5.2) to purify tRNAs. The tRNA fractions were pooled and tRNA was precipitated using the method described above and resuspended in $10 \mathrm{mM} \mathrm{NaOAc}$ ( $\mathrm{pH} 5.2$ ) for storage.

Acetyl-CoA hydrolysis. Proteins $(10 \mu \mathrm{M})$ were mixed with $100 \mu \mathrm{M}$ acetyl-CoA and tRNAs $(2 \mu \mathrm{M})$ or peptides $(2 \mu \mathrm{M})$ and incubated at $37^{\circ} \mathrm{C}$ for $20 \mathrm{~min}$. The reaction was applied to a $3 \mathrm{kDa}$ cutoff concentrator (EMD Millipore) to remove proteins. The flow through $(50 \mu \mathrm{L})$ was collected and transferred to a 96-well plate. Acetyl-CoA quantity was determined using an acetyl-CoA assay kit (Sigma) in accordance to manufacturer's instructions. An end-point assay was performed and the fluorescence intensity $\left(\lambda_{\text {Excitation }} 535 \mathrm{~nm} ; \lambda_{\text {Emission }} 587 \mathrm{~nm}\right)$ was measured using a plate reader (TECAN); $n=3$.

tRNA constructs and in vitro transcription and purification. All tRNA genes were amplified by PCR using genomic DNA from yeast as template and the primers that contain the complementary sequences to pUC19. Restriction free cloning method was performed to construct all amplified tRNA genes downstream of a T7 promoter. Downstream of every gene a BpiI cutting site was introduced, which is used to linearize the DNA for run off T7 RNA polymerase-driven in vitro transcription. The addition of CCA to the $3^{\prime}$-end of tRNA genes was performed by simple mutagenesis of the template construct. For in vitro transcription $(250 \mu \mathrm{L}$ 
reaction), the reaction contained linear DNA template $(20 \mathrm{ng}), 8 \mathrm{mM}$ of each NTPs (ATP, GTP, and UTP), $6 \mathrm{mM}$ of CTP and $2 \mathrm{mM}$ of Cy5-lableled CTP, T7 RNA polymerase $(0.5 \mathrm{~g} / \mathrm{L})$, RNasin $(0.025 \mathrm{~g} / \mathrm{L}), \mathrm{MgCl}_{2}(2 \sim 8 \mathrm{mM})$, pyrophosphatase $(1 \mathrm{U})$ and the reaction buffer ( $40 \mathrm{mM}$ Tris, $\mathrm{pH} 8.0,5 \mathrm{mM}$ DTT, $1 \mathrm{mM}$ spermidine, and $0.01 \%$ Triton X-100). All the ingredients were mixed at RT first followed by the incubation at $37^{\circ} \mathrm{C}$ for overnight. The next day, the RNase free DNase (2U) was added to the reaction for further incubation at $37^{\circ} \mathrm{C}$ for $15 \mathrm{~min}$. The reaction was then stopped by the addition of EDTA $(50 \mathrm{mM})$. The transcribed tRNA was purified using a DEAE column directly ${ }^{70}$ and eluted tRNA fractions were pooled and precipitated in $\mathrm{EtOH}$ at $-80^{\circ} \mathrm{C}$ for overnight. The tRNA containing solution was subjected to centrifuge at $20,000 \times g$ at $4{ }^{\circ} \mathrm{C}$ for $30 \mathrm{~min}$. The pellet was then washed in $70 \% \mathrm{EtOH}$, air-dried and re-dissolved in annealing buffer $(20 \mathrm{mM}$ HEPES, pH 7.5, $50 \mathrm{mM} \mathrm{NaCl}$, and $50 \mathrm{mM} \mathrm{KCl}$ ). Cy5-labeled tRNA was first renatured by heating in the annealing buffer at $80^{\circ} \mathrm{C}$ for $2 \mathrm{~min}$ and slowly cooled to $60{ }^{\circ} \mathrm{C}$ using a thermo-cycler. $\mathrm{MgCl}_{2}$ was added to tRNA and the procedure continued by cooling down to $25^{\circ} \mathrm{C}$. Annealed tRNAs were further purified by applying them to a gel filtration column $(\mathrm{S} 75,10 / 30)$ equilibrated in $20 \mathrm{mM}$ HEPES, pH 7.5, $150 \mathrm{mM} \mathrm{NaCl}, 5 \mathrm{mM}$ DTT, and $1 \mathrm{mM} \mathrm{MgCl}_{2}$. The purified tRNAs were then stored at $-80^{\circ} \mathrm{C}$.

EMSA. Cy5-labeled RNA oligos (ASL yeast tRNA ${ }^{\mathrm{Ser}_{\mathrm{UGA}}}$ 5'-GACAGACUUGAAAUCUGUU- $3^{\prime}$ and polyU $5^{\prime}$-UUUUUUUUU- $3^{\prime}$ ) were purchased from Sigma. Proteins were serially diluted, and Cy5-labeled RNA ( $\sim 1 \mathrm{ng} / \mathrm{lane})$ as well as Cy5-labeled tRNA were added for incubation at $25^{\circ} \mathrm{C}$ for $30 \mathrm{~min}$. The RNAprotein complex and free RNA were resolved by $6 \%$ native gel and separated by running for $6 \mathrm{~h}$ at $100 \mathrm{~V}$ at $4{ }^{\circ} \mathrm{C}$. The gels were scanned using Typhoon (GE Healthcare) and the images were analyzed and viewed by ImageQuant (GE Healthcare)

Microscale thermophoresis. MinElp3 (15 g/l) or DmcElp3 (10 g/l) were prepared in twofold serial dilutions in PCR tubes. The Cy5-labeled tRNA $(30 \mathrm{nM})$ or Cylabeled $\mathrm{H} 3$ peptide $(10 \mathrm{nM})$ was mixed with diluted proteins with equal amount for incubation at $25^{\circ} \mathrm{C}$ for $15 \mathrm{~min}$. The samples were subjected to premium capillary tubes and analyzed using MO. CONTROL (Nanotemper) with the temperature setting at $25^{\circ} \mathrm{C}$. The $K_{\mathrm{d}} \mathrm{s}$ were calculated based on triplicate measurement using MO. Affinity software (Nanotemper), $n=3$.

Isothermal titration calorimetry measurements. ITC was performed with Nano ITC 2G (TA Instruments). To measure the MinElp3 interaction with acetyl-CoA, histone 3 peptide or tubulin peptide, proteins were dialyzed against the ITC buffer (20 mM HEPES, pH 7.5, $150 \mathrm{mM} \mathrm{NaCl}$, and $1 \mathrm{mM} \beta$-mercaptoethanol) extensively overnight at $4{ }^{\circ} \mathrm{C}$. The reaction cell was filled with proteins $(10-12 \mu \mathrm{M})$; whereas, the ligands were prepared in the same ITC buffer at $2.47 \mathrm{mM}$ (except $\mathrm{H} 3$ was 1.6 $\mathrm{mM}$ ) and loaded into a dosing syringe. Each injection $(10 \mu \mathrm{l})$ was performed in 300 $s$ intervals. All experiments were conducted at $25^{\circ} \mathrm{C}$ with a stirring rate of $250 \mathrm{rpm}$. The results were analyzed using NanoAnalyze software (TA instruments).

\section{Data availability}

Data generated in this publication are available from corresponding author on reasonable request. The atomic coordinates and respective structure factors for DmcElp3-DCA (PDB ID 6IA6), MinElp3 11-19 (PDB ID 6IA8), MinElp3 11 -46 (PDB ID 6IAZ) and MinElp3 1-54 (PDB ID 6IAD) have been validated and deposited at the European Protein Data Bank. The source data underlying Figs 3, 4, Supplementary Fig. 1, 2, 3, 4, 5 and the numerical values of the graphs, including Figs 2, 3, 4 and Supplementary Fig. 3, 4, 5 and 6 are provided as a Source Data file.

Received: 24 August 2018 Accepted: 18 January 2019

Published online: 07 February 2019

\section{References}

1. Machnicka, M. A. et al. MODOMICS: a database of RNA modification pathways-2013 update. Nucleic Acids Res. 41, D262-D267 (2013).

2. Agris, P. F., Vendeix, F. A. \& Graham, W. D. tRNA's wobble decoding of the genome: 40 years of modification. J. Mol. Biol. 366, 1-13 (2007).

3. Grosjean, H., de Crecy-Lagard, V. \& Marck, C. Deciphering synonymous codons in the three domains of life: co-evolution with specific tRNA modification enzymes. FEBS Lett. 584, 252-264 (2010).

4. Helm, M. Post-transcriptional nucleotide modification and alternative folding of RNA. Nucleic Acids Res. 34, 721-733 (2006).

5. Wellner, K., Betat, H. \& Morl, M. A tRNA's fate is decided at its 3' end: collaborative actions of CCA-adding enzyme and RNases involved in tRNA processing and degradation. Biochim. Biophys. Acta Gene Regul. Mech. 1861, 433-441 (2018).
6. Agris, P. F. The importance of being modified: an unrealized code to RNA structure and function. RNA 21, 552-554 (2015).

7. Vare, V. Y., Eruysal, E. R., Narendran, A., Sarachan, K. L. \& Agris, P. F. Chemical and conformational diversity of modified nucleosides affects tRNA structure and function. Biomolecules 7, pii: E29 (2017).

8. Blanchet, S. et al. Deciphering the reading of the genetic code by near-cognate tRNA. Proc. Natl Acad. Sci. USA 115, 3018-3023 (2018).

9. Ranjan, N. \& Rodnina, M. V. Thio-Modification of tRNA at the Wobble position as regulator of the kinetics of decoding and translocation on the ribosome. J. Am. Chem. Soc. 139, 5857-5864 (2017).

10. Vendeix, F. A. et al. Human tRNA(Lys3)(UUU) is pre-structured by natural modifications for cognate and wobble codon binding through keto-enol tautomerism. J. Mol. Biol. 416, 467-485 (2012).

11. Rozov, A. et al. Novel base-pairing interactions at the tRNA wobble position crucial for accurate reading of the genetic code. Nat. Commun. 7, 10457 (2016).

12. Thommen, M., Holtkamp, W. \& Rodnina, M. V. Co-translational protein folding: progress and methods. Curr. Opin. Struct. Biol. 42, 83-89 (2017).

13. Rodnina, M. V., Fischer, N., Maracci, C. \& Stark, H. Ribosome dynamics during decoding. Philos. Trans. R Soc. Lond. B Biol. Sci. 372, pii: 20160182 (2017).

14. Zinshteyn, B. \& Gilbert, W. V. Loss of a conserved tRNA anticodon modification perturbs cellular signaling. PLoS Genet. 9, el003675 (2013).

15. Nedialkova, D. D. \& Leidel, S. A. Optimization of codon translation rates via tRNA modifications maintains proteome integrity. Cell 161, 1606-1618 (2015).

16. Klassen, R. et al. tRNA anticodon loop modifications ensure protein homeostasis and cell morphogenesis in yeast. Nucleic Acids Res. 44, 10946-10959 (2016).

17. Otero, G. et al. Elongator, a multisubunit component of a novel RNA polymerase II holoenzyme for transcriptional elongation. Mol. Cell 3, 109-118 (1999).

18. Winkler, G. S., Kristjuhan, A., Erdjument-Bromage, H., Tempst, P. \& Svejstrup, J. Q. Elongator is a histone $\mathrm{H} 3$ and $\mathrm{H} 4$ acetyltransferase important for normal histone acetylation levels in vivo. Proc. Natl Acad. Sci. USA 99, 3517-3522 (2002).

19. Huang, B., Johansson, M. J. O. \& Bystrom, A. S. An early step in wobble uridine tRNA modification requires the Elongator complex. RNA 11, 424-436 (2005).

20. Selvadurai, K., Wang, P., Seimetz, J. \& Huang, R. H. Archaeal Elp3 catalyzes tRNA wobble uridine modification at $\mathrm{C} 5$ via a radical mechanism. Nat. Chem. Biol. 10, 810-812 (2014).

21. Glatt, S. et al. Structural basis for tRNA modification by Elp3 from Dehalococcoides mccartyi. Nat. Struct. Mol. Biol. 23, 794-802 (2016).

22. Glatt, S., Seraphin, B. \& Muller, C. W. Elongator: transcriptional or translational regulator? Transcription 3, 273-276 (2012).

23. Chen, C., Huang, B., Anderson, J. T. \& Bystrom, A. S. Unexpected accumulation of $n \mathrm{~cm}(5) \mathrm{U}$ and $\mathrm{ncm}(5) \mathrm{S}(2)(\mathrm{U})$ in a trm 9 mutant suggests an additional step in the synthesis of $\mathrm{mcm}(5) \mathrm{U}$ and $\mathrm{mcm}(5) \mathrm{S}(2) \mathrm{U}$. PLoS ONE 6 , e20783 (2011)

24. Leidel, S. et al. Ubiquitin-related modifier Urm1 acts as a sulphur carrier in thiolation of eukaryotic transfer RNA. Nature 458, 228-232 (2009).

25. Johansson, M. J. O., Xu, F. \& Bystrom, A. S. Elongator a tRNA modifying complex that promotes efficient translational decoding. Biochim. Biophys. Acta 1861, 401-408 (2017).

26. Dauden, M. I. et al. Architecture of the yeast Elongator complex. EMBO Rep. 18, 264-279 (2017).

27. Setiaputra, D. T. et al. Molecular architecture of the yeast Elongator complex reveals an unexpected asymmetric subunit arrangement. EMBO Rep. 18, 280-291 (2017).

28. Glatt, S. et al. The Elongator subcomplex Elp456 is a hexameric RecA-like ATPase. Nat. Struct. Mol. Biol. 19, 314-320 (2012).

29. Esberg, A., Huang, B., Johansson, M. J. O. \& Bystrom, A. S. Elevated levels of two tRNA species bypass the requirement for elongator complex in transcription and exocytosis. Mol. Cell 24, 139-148 (2006).

30. Laguesse, S. et al. A dynamic unfolded protein response contributes to the control of cortical neurogenesis. Dev. Cell 35, 553-567 (2015).

31. Li, Q. et al. The Elongator complex interacts with PCNA and modulates transcriptional silencing and sensitivity to DNA damage agents. PLoS Genet. 5, e1000684 (2009)

32. Rahl, P. B., Chen, C. Z. \& Collins, R. N. Elplp, the yeast homolog of the FD disease syndrome protein, negatively regulates exocytosis independently of transcriptional elongation. Mol. Cell 17, 841-853 (2005).

33. Okada, Y., Yamagata, K., Hong, K., Wakayama, T. \& Zhang, Y. A role for the elongator complex in zygotic paternal genome demethylation. Nature 463, 554-558 (2010).

34. Miskiewicz, K. et al. ELP3 controls active zone morphology by acetylating the ELKS family member Bruchpilot. Neuron 72, 776-788 (2012). 
35. Laguesse, S. et al. Loss of Elp3 impairs the acetylation and distribution of connexin-43 in the developing cerebral cortex. Front. Cell Neurosci. 11, 122 (2017).

36. Ueki, Y., Shchepetkina, V. \& Lefcort, F. Retina-specific loss of Ikbkap/Elp1 causes mitochondrial dysfunction that leads to selective retinal ganglion cell degeneration in a mouse model of familial dysautonomia. Dis. Model Mech. 11, pii: dmm033746 (2018).

37. Torres, A. G., Batlle, E. \& Ribas de Pouplana, L. Role of tRNA modifications in human diseases. Trends Mol. Med. 20, 306-314 (2014).

38. Dauden, M. I., Jaciuk, M., Muller, C. W. \& Glatt, S. Structural asymmetry in the eukaryotic Elongator complex. FEBS Lett. 592, 502-515 (2017).

39. Rapino, F. et al. Codon-specific translation reprogramming promotes resistance to targeted therapy. Nature 558, 605-609 (2018).

40. Kojic, M. \& Wainwright, B. The many faces of Elongator in neurodevelopment and disease. Front. Mol. Neurosci. 9, 115 (2016).

41. Slaugenhaupt, S. A. et al. Tissue-specific expression of a splicing mutation in the IKBKAP gene causes familial dysautonomia. Am. J. Hum. Genet. 68, 598-605 (2001).

42. Bento-Abreu, A. et al. Elongator subunit 3 (ELP3) modifies ALS through tRNA modification. Hum. Mol. Genet. 27, 1276-1289 (2018).

43. Najmabadi, $H$. et al. Deep sequencing reveals 50 novel genes for recessive cognitive disorders. Nature 478, 57-63 (2011).

44. Kojic, M. et al. Elongator mutation in mice induces neurodegeneration and ataxia-like behavior. Nat. Commun. 9, 3195 (2018).

45. Paraskevopoulou, C., Fairhurst, S. A., Lowe, D. J., Brick, P. \& Onesti, S. The elongator subunit Elp3 contains a Fe4S4 cluster and binds Sadenosylmethionine. Mol. Microbiol. 59, 795-806 (2006).

46. Lin, T.-Y. \& Glatt, S. tRNA modification by elongator protein 3 (Elp3). in Encyclopedia of Inorganic and Bioinorganic Chemistry, vol. eibc2623 (ed. Scott, R. A.) (John Wiley \& Sons, Hoboken, NJ, 2018).

47. Schwalm, E. L., Grove, T. L., Booker, S. J. \& Boal, A. K. Crystallographic capture of a radical S-adenosylmethionine enzyme in the act of modifying tRNA. Science 352, 309-312 (2016).

48. Grove, T. L. et al. A radically different mechanism for S-adenosylmethioninedependent methyltransferases. Science 332, 604-607 (2011).

49. Trievel, R. C. et al. Crystal structure and mechanism of histone acetylation of the yeast GCN5 transcriptional coactivator. Proc. Natl Acad. Sci. USA 96, 8931-8936 (1999).

50. Tanner, K. G., Langer, M. R., Kim, Y. \& Denu, J. M. Kinetic mechanism of the histone acetyltransferase GCN5 from yeast. J. Biol. Chem. 275, 22048-22055 (2000).

51. Creppe, C. et al. Elongator controls the migration and differentiation of cortical neurons through acetylation of alpha-tubulin. Cell 136, 551-564 (2009).

52. Howes, S. C., Alushin, G. M., Shida, T., Nachury, M. V. \& Nogales, E. Effects of tubulin acetylation and tubulin acetyltransferase binding on microtubule structure. Mol. Biol. Cell 25, 257-266 (2014).

53. Kuo, Y. M. \& Andrews, A. J. Quantitating the specificity and selectivity of Gcn5-mediated acetylation of histone H3. PLoS ONE 8, e54896 (2013).

54. Imoberdorf, R. M., Topalidou, I. \& Strubin, M. A role for gcn5-mediated global histone acetylation in transcriptional regulation. Mol. Cell Biol. 26, 1610-1616 (2006).

55. Amlacher, S. et al. Insight into structure and assembly of the nuclear pore complex by utilizing the genome of a eukaryotic thermophile. Cell 146, 277-289 (2011).

56. Glatt, S. et al. Structure of the Kti11/Kti13 heterodimer and its double role in modifications of tRNA and eukaryotic elongation factor 2. Structure 23, 149-160 (2015).

57. Mehlgarten, C. et al. Use of a yeast tRNase killer toxin to diagnose Kti12 motifs required for tRNA modification by Elongator. Toxins 9, pii: E272 (2017).

58. van Tran, N. et al. Evolutionary insights into Trm112-methyltransferase holoenzymes involved in translation between archaea and eukaryotes. Nucleic Acids Res. 46, 8483-8499 (2018).

59. Agris, P. F. Decoding the genome: a modified view. Nucleic Acids Res. 32, 223-238 (2004).

60. Sokolowski, M., Klassen, R., Bruch, A., Schaffrath, R. \& Glatt, S. Cooperativity between different tRNA modifications and their modification pathways. Biochim. Biophys. Acta 1861, 409-418 (2018).

61. Kabsch, W. Xds. Acta Crystallogr. D Biol. Crystallogr. 66, 125-132 (2010).

62. McCoy, A. J. et al. Phaser crystallographic software. J. Appl. Crystallogr. 40, 658-674 (2007).
63. Adams, P. D. et al. PHENIX: building new software for automated crystallographic structure determination. Acta Crystallogr. D Biol. Crystallogr. 58, 1948-1954 (2002)

64. Davis, I. W. et al. MolProbity: all-atom contacts and structure validation for proteins and nucleic acids. Nucleic Acids Res. 35, W375-W383 (2007).

65. DeLano, W. L. The PyMOL Molecular Graphics System. (2002)

66. Emsley, P. \& Cowtan, K. Coot: model-building tools for molecular graphics. Acta Crystallogr. D Biol. Crystallogr. 60, 2126-2132 (2004).

67. Hodel, A., Kim, S. H. \& Brunger, A. T. Model bias in macromolecular crystalstructures. Acta Crystallogr. Sect. A 48, 851-858 (1992).

68. Chan, W. C. \& White, P. D. Fmoc solid phase peptide synthesis: a practical approach xxiv, (346. Oxford University Press, New York, 2000).

69. Klassen, R. \& Schaffrath, R. Role of Pseudouridine formation by Deg1 for functionality of two glutamine isoacceptor tRNAs. Biomolecules 7, pii: E8 (2017).

70. Easton, L. E., Shibata, Y. \& Lukavsky, P. J. Rapid, nondenaturing RNA purification using weak anion-exchange fast performance liquid chromatography. RNA 16, 647-653 (2010).

\section{Acknowledgements}

We thank Barbara Kościelniak-Merak and Rościsław Krutyhołowa for help with tRNA and protein production. We thank Karin D Breunig, Jonathan Heddle, and Przemyslaw Grudnik for critical comments on the manuscript. We thank Przemyslaw Grudnik and beamline staff at beamlines 14.1 (BESSY) and P11 (PETRAIII) for support during data collection. We thank Raffael Schaffrath and Alex Hammermeister for yeast strains. We thank the MCB structural biology core facility (supported by the TEAM TECH CORE FACILITY/2017-4/6 grant from Foundation for Polish Science) for providing instruments and support. In addition, this work was supported by the OPUS10 grant (UMO2015/19/B/NZ1/00343; K.Z. and S.G.) from the National Science Centre and the Homing program (Homing/2016-2/14; T.-Y.L. and N.E.H.A.) from the Foundation for Polish Science.

\section{Author contributions}

T.-Y.L. performed biochemical, biophysical, and crystallographic analyses; T.-Y.L. purified proteins with the help of N.E.H.A. and K.Z.; N.E.H.A. characterized Elp3 mutants on biophysical analyses; A.C.-G. produced and purified tRNAs; M.J.-R. performed the ITC measurements and analysis; J.R. synthesized and purified lysine-containing peptides; T.-Y.L. and S.G. designed experiments, collected crystallographic data, refined structures, analyzed the results, prepared figures, and wrote the manuscript, with the input from all other authors.

\section{Additional information}

Supplementary Information accompanies this paper at https://doi.org/10.1038/s41467019-08579-2.

Competing interests: The authors declare no competing interests.

Reprints and permission information is available online at http://npg.nature.com/ reprintsandpermissions/

Journal peer review information: Nature Communications thanks the anonymous reviewers for their contribution to the peer review of this work.

Publisher's note: Springer Nature remains neutral with regard to jurisdictional claims in published maps and institutional affiliations.

Open Access This article is licensed under a Creative Commons Attribution 4.0 International License, which permits use, sharing, adaptation, distribution and reproduction in any medium or format, as long as you give appropriate credit to the original author(s) and the source, provide a link to the Creative Commons license, and indicate if changes were made. The images or other third party material in this article are included in the article's Creative Commons license, unless indicated otherwise in a credit line to the material. If material is not included in the article's Creative Commons license and your intended use is not permitted by statutory regulation or exceeds the permitted use, you will need to obtain permission directly from the copyright holder. To view a copy of this license, visit http://creativecommons.org/ licenses/by/4.0/.

(C) The Author(s) 2019 